TRANSACTIONS OF THE

AMERICAN MATHEMATICAL SOCIETY

Volume 364, Number 7, July 2012, Pages 3585-3607

S 0002-9947(2012)05641-8

Article electronically published on March 7, 2012

\title{
RESTRICTED BERGMAN KERNEL ASYMPTOTICS
}

\author{
TOMOYUKI HISAMOTO
}

\begin{abstract}
In this paper, we investigate a restricted version of Bergman kernels for high powers of a big line bundle over a smooth projective variety. The geometric meaning of the leading term is specified. As a byproduct, we derive some integral representations for the restricted volume.
\end{abstract}

\section{INTRODUCTION}

The subjects discussed in this paper originate from the extension problem. Let $L$ be a big line bundle on a smooth complex projective variety $X$ and $Z \subseteq X$ a subvariety. Denote by $\iota: Z \hookrightarrow X$ the inclusion map. We always use this notation unless specifically noted. It is important to know how many sections of $\left.L\right|_{Z}$ are extended to the ambient space $X$. We can expect to get more such sections taking high tensor powers of $L$, thus we are led to consider the spaces of sections

$$
H^{0}(X \mid Z, \mathcal{O}(m L)):=\operatorname{Im}\left[\iota^{*}: H^{0}(X, \mathcal{O}(m L)) \rightarrow H^{0}(Z, \mathcal{O}(m L))\right] .
$$

The restricted volume

$$
\operatorname{Vol}_{X \mid Z}(L):=\limsup _{m \rightarrow \infty} \frac{\operatorname{dim} H^{0}(X \mid Z, \mathcal{O}(m L))}{m^{p} / p !}
$$

measures the asymptotic growth of these spaces. Here $p$ denotes the complex dimension of $Z$. The notion of the restricted volume first appeared in Tsuji's paper Tsu06 (see also HM06, Tak06], ELMNP09]). In this paper, we investigate a local version of the restricted volume.

Definition 1.1. Let $h_{L}$ be a smooth Hermitian metric on $L, \varphi \in C^{\infty}(X ; \mathbb{R})$ a smooth weight, and $d \mu$ a volume form on $Z$. Then for any positive integer $m$, the restricted Bergman kernel of $\left(Z, m L, h_{L}^{m} e^{-m \varphi}, d \mu\right)$ is defined as follows:

$$
B_{X \mid Z}(m \varphi):=\left|s_{m, 1}\right|_{m \varphi}^{2}+\cdots+\left|s_{m, N(m)}\right|_{m \varphi}^{2} .
$$

Here $\left\{s_{m, 1}, \ldots, s_{m, N(m)}\right\}$ is a complete orthonormal system of $H^{0}(X \mid Z, \mathcal{O}(m L))$ with respect to the norm

$$
\begin{gathered}
\|s\|_{m \varphi}^{2}:=\int_{Z}|s|_{m \varphi}^{2} d \mu, \\
|s|_{m \varphi}^{2}:=\iota^{*} h_{L}^{m}(s, s) e^{-m \iota^{*} \varphi} .
\end{gathered}
$$

By definition, $B_{X \mid Z}(m \varphi)$ is a smooth function on $Z$ and

$$
\int_{Z} B_{X \mid Z}(m \varphi) d \mu=\operatorname{dim} H^{0}(X \mid Z, \mathcal{O}(m L)) .
$$

Received by the editors June 22, 2010.

2010 Mathematics Subject Classification. Primary 32A25; Secondary 32L10, 32W20.

Key words and phrases. Bergman kernel, extension theorem, Monge-Ampère operator.

(C)2012 American Mathematical Society 
In fact $B_{X \mid Z}(m \varphi)$ tells not only the dimension but rather deeper information of the space of sections. The study of the asymptotic behavior of $B_{X \mid Z}(m \varphi)$ is itself an important problem in complex geometry.

In this paper we closely examine the leading term of $B_{X \mid Z}(m \varphi)$. If $L$ is ample and the metric $h_{L} e^{-\varphi}$ has positive curvature, then by the Serre vanishing theorem the problem is reduced to the case $Z=X$. In this case, Tian's classical result ([Tian90]) gives the complete answer. For general $L$ and $h_{L}$, Berman first treated the case $Z=X$ in Ber09. And in that paper, he also mentioned the restricted case without proof. Without the assumption of curvature positivity, the effect of the subvariety cannot be ignored. We give a complete picture in the restricted case and specify the limit of $m^{-p} B_{X \mid Z}(m \varphi)$. Our study can be seen as a local version of the restricted Fujita-type approximation (Theorem 3.19). As a result, a localization of $\mathrm{Vol}_{X \mid Z}(L)$ is given.

To state our results, we need some notion which arises in non-positive curvature case. First denote by $\mathbb{B}_{+}(L) \subsetneq X$ the augmented base locus (see Laz04, Definition 10.3.2]). This is actually an algebraic subset of $X$, and $L$ is ample precisely if $\mathbb{B}_{+}(L)=\emptyset$. Secondly, we denote by $P_{X \mid Z} \varphi$ the equilibrium weight associated to $\varphi$ (see Definition 3.1). Let $\theta=-d d^{c} \log h_{L}$ be the Chern curvature of $h_{L}$, then $\theta+d d^{c} P_{X \mid Z} \varphi$ defines a positive current on $Z$, and $P_{X \mid Z} \varphi=\varphi$ holds if $\theta+d d^{c} \varphi$ is positive. Roughly speaking, $P_{X \mid Z} \varphi$ is the best $\theta$-plurisubharmonic function on $Z$ approximating $\varphi$. Further, one can measure the rest of the positivity of $\theta+d d^{c} \varphi$ by $\operatorname{MA}\left(P_{X \mid Z} \varphi\right):=\left\langle\left(\theta+d d^{c} P_{X \mid Z} \varphi\right)^{p}\right\rangle$, the non-pluripolar Monge-Ampère (MA) product of $P_{X \mid Z} \varphi$ (see Definition 2.4).

Theorem 1.2. Assume $Z$ is smooth and $Z \nsubseteq \mathbb{B}_{+}(L)$. Then the convergence

$$
\frac{B_{X \mid Z}(m \varphi)}{m^{p} / p !} d \mu \rightarrow \operatorname{MA}\left(P_{X \mid Z} \varphi\right)
$$

holds in the sense of currents.

As byproducts of our investigation of restricted Bergman kernel asymptotics, we can get several integral representations of restricted volumes (discussed in Section 4). For instance, we have the following.

Theorem 1.3. In the situation of Theorem 1.2, the following holds.

$$
\begin{aligned}
\operatorname{Vol}_{X \mid Z}(L) & =\int_{Z} \operatorname{MA}\left(P_{X \mid Z} \varphi\right)=\int_{Z} \operatorname{MA}\left(\iota^{*} P_{X} \varphi\right) \\
& =\sup _{T} \int_{Z}\left\langle\left(\iota^{*} T\right)^{p}\right\rangle=\int_{Z}\left\langle\left(\iota^{*} T_{\min }\right)^{p}\right\rangle,
\end{aligned}
$$

where $T$ runs through all the closed positive currents in $c_{1}(L)$, with small unbounded loci not contained in $\iota(Z)$. We denote by $T_{\min }$ a minimum singular closed positive current in $c_{1}(L)$.

These formulas can be seen as generalizations of the main result of Bou02]. If $L$ is ample, $\operatorname{Vol}_{X \mid Z}(L)$ equals the intersection number $\left(L^{p} . Z\right)$ which plays an important role in many geometric questions. But for general line bundles, these intersection numbers do not work well to describe function-theoretic properties of $L$. Our results indicate that $\operatorname{Vol}_{X \mid Z}(L)$ is the natural generalization of $\left(L^{p} . Z\right)$ for general line bundles. 
Let us explain the point of our proof of Theorem 1.2. We basically follow Berman's approach, but there are two difficulties in the restricted case. First, to deal with general subvarieties, we need a variant of $L^{2}$-extension theorems. The desired extension theorem is the following, which is proved in Section 5 (Theorem 1.4).

Theorem 1.4. Let $X$ be a smooth projective variety, $Z \subseteq X$ a smooth subvariety, $\omega$ a fixed Kähler form, and $E \rightarrow X$ a holomorphic vector bundle with a smooth Hermitian metric $h_{E}$. Then there exist constants $N=N\left(Z, X, h_{E}, \omega\right)$ and $C=$ $C(Z, X)>0$ such that the following holds.

Let $L \rightarrow X$ be a holomorphic line bundle with a singular Hermitian metric $h_{L} e^{-\varphi}$ such that its Chern curvature satisfies

$$
\theta+d d^{c} \varphi \geqslant N \omega
$$

Then for any section $s \in H^{0}(Z, \mathcal{O}(E \otimes L))$ with

$$
\int_{Z}|s|^{2} e^{-\varphi} d V_{\omega, Z}<+\infty
$$

there exists a section $\widetilde{s} \in H^{0}(X, \mathcal{O}(E \otimes L))$ such that $\left.\widetilde{s}\right|_{Z}=s$ and

$$
\int_{X}|\widetilde{s}|^{2} e^{-\varphi} d V_{\omega, X} \leqslant C \int_{Z}|s|^{2} e^{-\varphi} d V_{\omega, Z}
$$

holds.

Remark 1.5. It is natural to expect that Theorem 1.4 holds even if $Z$ has some mild singularities. But it seems to be unknown.

Theorem 1.4 can be derived from Theorem 4 of Ohs01 by a standard approximation technique. See also Kim10. It seems most likely that a slight change of the proof of Theorem 4.2 of [Kim10] can yield Theorem 1.4. At any rate we give a self-contained proof in Section 5, as a courtesy to the reader. Theorem 5.1 in the present paper corresponds to Theorem 4 in Ohs01] (but the situations in the two theorems are slightly different).

Theorem 1.4 is used in two critical steps. One step is to show the regularity of the restricted equilibrium weight (Theorem 3.5), and the other is to show a lower bound of restricted Bergman kernels (Theorem 3.15). Second, we only have a weak lower bound in the restricted case since it becomes harder to estimate the lower bound of the Bergman kernels precisely as Ber09. We avoid this difficulty by using a proof of the restricted version of the Fujita-type approximation theorem. Note that a part of this strategy already appeared in [BB10. We elaborate upon this strategy using a weak lower bound and the comparison theorem for the MongeAmpère operator. From this, one can first get an integral representation of the restricted volume and then deduce Theorem [1.2. Compared with Ber09] in the case $Z=X$, our proof of Theorem 1.2 is rather geometric thanks to the Fujita-type approximation. On the other hand, the convergence result obtained in this paper is weaker than that of Ber09]. It seems to be unknown whether convergence in a strict sense holds in the restricted case.

\section{Monge-Ampère operator}

We briefly review the definition of the Monge-Ampère operator in this section. Fix a closed real smooth $(1,1)$-form $\theta$ defined on $X$. An $L_{\text {loc }}^{1}$-function $\psi$ in $X$ is 
called $\theta$-plurisubharmonic ( $\theta$-psh for short) when the associated current $\theta+d d^{c} \psi$ is positive (in the sense of currents). A function which is $\theta$-psh for some $\theta$ is called quasi-plurisubharmonic (quasi-psh for short). It is known that $\psi$ automatically becomes upper-semicontinuous by this condition. We denote the set of all $\theta$-psh functions by $\operatorname{PSH}(X, \theta)$. In this paper we are mainly interested in $\theta$ defined as $\theta=-d d^{c} \log h_{L}$, but this notion is in fact valid for an arbitrary $\theta$.

Let $n$ be the dimension of $X$. The Monge-Ampère operator should be defined as

$$
\psi \mapsto \mathrm{MA}(\psi):=\left(\theta+d d^{c} \psi\right)^{n},
$$

but for general $\psi$, this is nonsense. The celebrated result of Bedford and Taylor ([BT76] $)$ tells us that the right-hand side can be defined as a current for $\psi$ at least in the class $L_{\text {loc }}^{\infty} \cap \operatorname{PSH}(X, \theta)$. That is, by induction on the exponent $q=1,2, \ldots, n$, it can be defined as

$$
\int_{X}\left(\theta+d d^{c} \psi\right)^{q} \wedge \eta:=\int_{X}\left(\theta+d d^{c} \psi\right)^{q-1} \wedge(\tau+\psi) d d^{c} \eta
$$

for each test form $\eta \in C_{0}^{\infty}\left(X, \bigwedge^{n-q, n-q} T_{X}^{*}\right)$. Here $\int_{X}$ denotes the canonical pairing of currents and test forms, and $\tau$ denotes a local $d d^{c}$-potential of $\theta$. This is indeed well defined and defines a closed positive current, because $\tau+\psi$ is a bounded Borel function and $\left(\theta+d d^{c} \psi\right)^{q-1}$ has measure coefficients by the induction hypothesis and by the fact that any closed positive current has measure coefficients. Bedford and Taylor's Monge-Ampère products have useful continuity properties:

Proposition 2.1.

$$
\left(\theta+d d^{c} \psi_{k}\right)^{n} \rightarrow\left(\theta+d d^{c} \psi\right) \quad \text { in the sense of currents }
$$

for any sequence of $\theta$-psh functions which satisfies one of the following conditions:

(1) $\psi_{k} \searrow \psi$ pointwise in $X$;

(2) $\psi_{k} \nearrow \psi$ for almost every point in $X$; or

(3) $\psi_{k} \rightarrow \psi$ uniformly in any compact subset of $X$.

It is still necessary to consider unbounded $\theta$-psh functions. On the other hand, for our purposes of investigating asymptotic behaviors of Bergman kernels, it is sufficient to deal with some special class of unbounded $\theta$-psh functions, and we can omit a part of the contribution of unbounded loci.

Definition 2.2. A $\theta$-psh function $\psi$ is said to have a small unbounded locus if the pluripolar set $\psi^{-1}(-\infty)$ is contained in some closed proper algebraic subset $S \subsetneq X$.

A quasi-psh function $\psi$ on $X$ is said to have algebraic singularities, if it can be locally written as

$$
\psi=c \cdot \log \left(\left|f_{1}\right|^{2}+\cdots+\left|f_{N}\right|^{2}\right)+u
$$

for some $c \in \mathbb{Q} \geqslant 0$, non-zero regular functions $f_{i}(1 \leqslant i \leqslant N)$, and a smooth function $u$. Every $\theta$-psh function with algebraic singularities has a small unbounded locus. If we assume the subvariety $Z$ is smooth, $\iota^{*} \varphi+m^{-1} \log B_{X \mid Z}(m \varphi)$ gives the typical example of $\iota^{*} \theta$-psh function with algebraic singularities.

Definition 2.4. For a $\theta$-psh function $\psi$ on $X$ with a small unbounded locus, MA $(\psi)$ is defined to be

$$
\left\langle\left(\theta+d d^{c} \psi\right)^{n}\right\rangle:=\text { the zero extension of }\left(\theta+d d^{c} \psi\right)^{n} .
$$

Note that the coefficient of $\left(\theta+d d^{c} \psi\right)^{n}$ is well defined as a measure on $X \backslash S$. 
MA $(\psi)$ actually defines a closed positive current on $X$ by the famous Skoda's extension theorem. In particular, it has a finite mass on $X$. For a proof, see [BEGZ10, section 1].

Remark 2.5. In that paper, the non-pluripolar Monge-Ampère product was defined in fact for general $\theta$-psh functions on a compact Kähler manifold. Note that this choice of ways to define the Monge-Ampère operator causes MA $(\psi)$ to have no mass on any pluripolar set and so ignores some of the singularities of $\psi$. For this reason, $\left\langle\left(\theta+d d^{c} \psi\right)^{n}\right\rangle$ no longer has continuity property with respect to $\psi$.

We recall the fundamental fact established in [BEGZ10] which states that the less singular $\theta$-psh function has the larger Monge-Ampère mass. Recall that given two $\theta$-psh $\psi$ and $\psi^{\prime}, \psi$ is said to be less singular than $\psi^{\prime}$ if there exists a constant $C>0$ such that $\psi^{\prime} \leqslant \psi+C$ in $X$. We say that a $\theta$-psh function is minimal singular if it is minimal with respect to this partial order. When $\psi$ is less singular than $\psi^{\prime}$ and $\psi^{\prime}$ is less singular than $\psi$, we say that the two functions are equivalent with respect to singularities. This defines an equivalence relation in $\operatorname{PSH}(X, \theta)$. When $\theta \in c_{1}(L)$, any minimal singular $\theta$-psh function $\psi$ has a small unbounded locus. In fact, $\psi^{-1}(-\infty) \subseteq \mathbb{B}_{+}(L)$ holds.

Theorem 2.6 ([BEGZ10, Theorem 1.16]). If $\psi, \psi^{\prime}$ are $\theta$-psh functions with small unbounded loci such that $\psi$ is less singular than $\psi^{\prime}$, then

$$
\int_{X} \operatorname{MA}\left(\psi^{\prime}\right) \leqslant \int_{X} \operatorname{MA}(\psi)
$$

holds.

Remark 2.7. It is unknown whether Theorem 2.6 holds for general $\theta$-psh functions.

The notion of types of $\theta$-psh functions with respect to singularities, explained in this subsection, are in fact determined by the closed positive currents $T:=\theta+d d^{c} \psi$. Namely, a closed positive $(1,1)$-current $T \in \alpha$ is said to have a small unbounded locus if it can be written $T=\theta+d d^{c} \psi$ with some $\psi$ which has a small bounded locus. Closed positive $(1,1)$-currents with algebraic singularities and those with minimal singularities can be defined in the same manner.

\section{Restricted Bergman kernel asymptotics}

3.1. Restricted equilibrium weight. In this subsection, we introduce the notion of the restricted equilibrium weight and discuss its properties, which we will use later to study asymptotics of restricted Bergman kernels. Unless otherwise stated, we fix a big line bundle $L$ on a smooth projective variety $X$ and a smooth metric $h_{L}$. Let $\theta:=-d d^{c} \log h_{L}$ be the Chern curvature form. Given a subvariety $Z$ of $X$, there exists a canonical way to associate any smooth function to the $\theta$-psh function on $Z$.

Definition 3.1. For a smooth weight $\varphi \in C^{\infty}(X ; \mathbb{R})$ and a subvariety $Z \subseteq X$, the restricted equilibrium weight $P_{X \mid Z} \varphi$ is a function on $Z$ defined as

$$
P_{X \mid Z} \varphi(z):=\sup ^{*}\left\{\begin{array}{l|l}
\iota^{*} \psi(z) & \begin{array}{c}
\psi \in \operatorname{PSH}(X, \theta) \\
\text { with } \iota^{*} \psi \leqslant \iota^{*} \varphi \text { on } Z
\end{array}
\end{array}\right\}
$$

for $z \in Z$. Here $\iota: Z \hookrightarrow X$ denotes the inclusion map. If there is no $\psi$ as above, $P_{X \mid Z} \varphi \equiv-\infty$ by definition. 
In the special case when $Z=X$, we use the notation $P_{X}$ instead of $P_{X \mid X}$ as in [BB10. The symbol sup* that appeared in the above definition means

$$
\sup _{\alpha}^{*} f_{\alpha}(z):=\limsup _{w \rightarrow z}\left(\sup _{\alpha} f_{\alpha}(w)\right) \text {, }
$$

which is called the regularized upper envelope for a family of functions $\left\{f_{\alpha}\right\}_{\alpha}$. It is easily seen that $\iota^{*} P_{X} \varphi \leqslant P_{X \mid Z} \varphi \leqslant P_{Z} \iota^{*} \varphi$ holds. By a classical result of Choquet (see, e.g., Kli91, Lemma 2.3.4]) and by the definition of $P_{X \mid Z} \varphi$, we get the following.

Lemma 3.3. Assume that $P_{X \mid Z} \varphi$ is not identically infinity on $Z$. Then there exists a countable non-decreasing family of $\theta$-psh functions $\left\{\psi_{k}\right\}_{k}(k=1,2, \ldots)$ such that $\iota^{*} \psi_{k} \nearrow P_{X \mid Z} \varphi$ a.e., otherwise, $P_{X \mid Z} \varphi \equiv-\infty$. In particular, $P_{X \mid Z} \varphi \in \operatorname{PSH}\left(Z, \iota^{*} \theta\right)$ unless $P_{X \mid Z} \varphi \equiv-\infty$.

Now assume that $Z$ is smooth and that $\iota(Z) \nsubseteq \mathbb{B}_{+}(L)$. Then $P_{X \mid Z} \varphi$ has a small unbounded locus contained in $\iota^{-1}\left(\mathbb{B}_{+}(L)\right)$. Then it follows that the Monge-Ampère mass of $P_{X \mid Z} \varphi$ can be defined as

$$
\int_{Z} \operatorname{MA}\left(P_{X \mid Z} \varphi\right):=\int_{Z}\left\langle\left(\iota^{*} \theta+d d^{c} P_{X \mid Z} \varphi\right)^{p}\right\rangle=\int_{Z \backslash \iota^{-1}\left(\mathbb{B}_{+}(L)\right)}\left(\iota^{*} \theta+d d^{c} P_{X \mid Z} \varphi\right)^{p} .
$$

The following is a consequence of Theorem [2.6. and it enables us to substitute $\operatorname{MA}\left(\iota^{*} P_{X} \varphi\right)$ for $\operatorname{MA}\left(P_{X \mid Z} \varphi\right)$ to estimate the lower bound of the restricted Bergman kernels. This is the starting point of our strategy to prove Theorem 1.2 ,

Theorem 3.4. Assume that $Z$ is smooth and that $\iota(Z) \nsubseteq \mathbb{B}_{+}(L)$. Then it holds that

$$
\int_{Z} \operatorname{MA}\left(P_{X \mid Z} \varphi\right)=\int_{Z} \operatorname{MA}\left(\iota^{*} P_{X} \varphi\right) .
$$

Proof. By Theorem 2.6. we only have to show the first equality. The one side inequality $\geqslant$ is also an immediate consequence of Theorem 2.6. Since we may take $\psi_{k}$ minimal singular in Lemma 3.3 (by exchanging $\psi_{k}$ by $\max \left\{\psi_{k}, P_{X} \varphi\right\}$ ),

$$
\int_{Z} \operatorname{MA}\left(\iota^{*} P_{X} \varphi\right)=\int_{Z} \operatorname{MA}\left(\iota^{*} \psi_{k}\right)
$$

holds by Theorem 2.6. On the other hand, since

$$
\left(\iota^{*} \theta+d d^{c} \iota^{*} \psi_{k}\right)^{p} \rightarrow\left(\iota^{*} \theta+d d^{c} P_{X \mid Z \varphi} \varphi\right)^{p} \quad \text { on } Z \backslash \iota^{-1}\left(\mathbb{B}_{+}(L)\right)
$$

by the continuity property of the Monge-Ampère operator, we have

$$
\liminf _{k \rightarrow \infty} \int_{Z} \operatorname{MA}\left(\iota^{*} \psi_{k}\right) \geqslant \int_{Z} \operatorname{MA}\left(P_{X \mid Z} \varphi\right) .
$$

Therefore,

$$
\int_{Z} \operatorname{MA}\left(\iota^{*} P_{X} \varphi\right) \geqslant \int_{Z} \operatorname{MA}\left(P_{X \mid Z} \varphi\right) .
$$

The next theorem is a key ingredient for representing $\operatorname{MA}\left(P_{X \mid Z} \varphi\right)$ explicitly by $\varphi$. It states that the gradient of $P_{X \mid Z} \varphi$ is locally Lipschitz on $Z \backslash \mathbb{B}_{+}(L)$.

Theorem 3.5. Assume that $Z$ is smooth and $\iota(Z) \nsubseteq \mathbb{B}_{+}(L)$. Then $P_{X \mid Z} \varphi$ has Lipschitz continuous first derivatives outside of $\iota^{-1}\left(\mathbb{B}_{+}(L)\right)$. Namely,

$$
P_{X \mid Z} \varphi \in C^{1,1}\left(Z \backslash \iota^{-1}\left(\mathbb{B}_{+}(L)\right)\right) .
$$


Moreover,

$\left(\iota^{*} \theta+d d^{c} P_{X \mid Z} \varphi\right)^{p}=\left(\iota^{*} \theta+d d^{c} \iota^{*} \varphi\right)^{p} \quad$ in the set $\quad\left\{P_{X \mid Z} \varphi=\iota^{*} \varphi\right\} \backslash \iota^{-1}\left(\mathbb{B}_{+}(L)\right)$

a.e. with respect to $d \mu$.

Proof. The proof is almost the same as the $Z=X$ case in Ber09 except that in the restricted case we need the Ohsawa-Takegoshi-type $L^{2}$-extension theorem for an arbitrary smooth subvariety (Theorem 1.4). We sketch the proof and omit the detail.

Let $Y$ be the total space of the dual line bundle $L^{*}$, identifying the base $X$ with its embedding as the zero section in $Y$, and let $\pi: Y \rightarrow X$ be the projection map. Given $\psi \in \operatorname{PSH}(X, \theta)$, one can associate a psh function $\chi_{\psi}$ defined on $Y$, as follows:

$$
\chi_{\psi}(x, w):=\log |w|_{h_{L}^{-1}}^{2}+\psi(x) \quad\left(x \in X, w \in L_{x}\right) .
$$

Berman's original argument is modeled on the proof of Bedford-Taylor for $C^{1,1}$ regularity of the solution of the Dirichlet problem for the complex Monge-Ampère equation in the unit-ball in $\mathbb{C}^{n}$. As opposed to the unit ball, $X$ has no global holomorphic vector fields. But one can reduce the regularity problem of $P_{X} \varphi$ on $X$ to a problem of $\chi_{P_{X} \varphi}$ on $Y$, where sufficiently many vector fields exist. This argument is still valid in the restricted case once one can construct the suitable vector fields on $\pi^{-1}(Z)$ extended to $Y$.

For a proof, it is enough to show the regularity of $\chi_{P_{X} \varphi}$ at any given point $y_{0} \in \pi^{-1}\left(Z \backslash \mathbb{B}_{+}(L)\right) \backslash Z$. By Kodaira's lemma, there exists an effective divisor $E$ on $X$ such that $y_{0} \notin \pi^{-1}(\operatorname{Supp} E)$ and $m L=A+E$ hold with some positive integer $m$ and ample $\mathbb{Z}$-divisor $A$. We may assume $m=1$ for the proof of Theorem 3.5 since $m P_{X \mid Z} \varphi=P_{X \mid Z}(m \varphi)$ holds. By this decomposition, we can construct some $\psi_{0}=\psi_{A}+\psi_{E}, \theta_{L}=\theta_{A}+\theta_{E}$ such that $\theta_{A}+d d^{c} \psi_{A}>0$ is smooth and $\theta_{E}+d d^{c} \psi_{E} \geqslant 0$ has singularities only on $E$. Indeed, it is enough to set $\theta_{E}:=$ $-d d^{c} \log h_{E, \alpha}, \psi_{E}:=\log \left|f_{\alpha}\right|^{2}+\log h_{E, \alpha}$ for some smooth metric $h_{E}$ and system of local equations $\left\{f_{\alpha}\right\}$.

Lemma 3.6. There exist holomorphic vector fields $V_{1}, \ldots, V_{p+1}$ on $\pi^{-1}(Z)$ satisfying the following properties:

(1) $V_{1}, \ldots, V_{p+1}$ is linearly independent at $y_{0}$.

(2) There exist holomorphic vector fields $\widetilde{V}_{1}, \ldots, \widetilde{V}_{p+1}$ on $Y$ such that $\left.\widetilde{V}_{i}\right|_{\pi^{-1}(Z)}$ $=V_{i}(1 \leqslant i \leqslant p+1)$.

(3) For any fixed $k \in \mathbb{N}, \widetilde{V}_{i}(1 \leqslant i \leqslant p+1)$ can be chosen to have zeros of order at least $k$ along $X$ and $\pi^{-1}(\operatorname{Supp} E)$. To be precise,

$$
\left|\widetilde{V}_{i}\right| \leqslant C(k) \cdot|w|^{k}, \quad\left|\widetilde{V}_{i}\right| \leqslant C(k) \cdot\left|f_{\alpha}(z)\right|^{k}
$$

hold locally in the set $\left\{\chi_{\psi_{0}} \leqslant 1\right\}$ for some constant $C(k)$ depending on $k$.

Proof. Let $\widehat{Y}:=\mathbb{P}(\mathcal{O}(-L) \oplus \mathcal{O})$ be the Zariski closure of $Y$. Consider the line bundle $\pi^{*} L^{k_{0}} \otimes H_{\mathbb{P}(\mathcal{O}(-L) \oplus \mathcal{O})}$ on $\widehat{Y}$ and its metric

$$
h_{k_{0}, \alpha}:=\pi^{*} h_{L, \alpha}^{k_{0}}+\log \left(1+e^{\chi \varphi}\right)
$$

with weight

$$
\psi_{k_{0}}:=\pi^{*}\left(k_{0}\left(\psi_{A}+\left(1+k_{0}^{-1 / 2}\right) \psi_{E}\right)\right)
$$


Here $H_{\mathbb{P}(\mathcal{O}(-L) \oplus \mathcal{O})}$ denotes the fiberwise hyperplane bundle. For $w$-direction, $\log \left(1+e^{\chi_{\varphi}}\right)$ has strictly positive curvature and for the $x$-direction, $\psi_{A}+(1+$ $\left.k_{0}^{-1 / 2}\right) \psi_{E}$ is $\theta_{L}$ strictly positive if we take $k_{0}$ sufficiently large. Thus $h_{k_{0}} e^{-\psi_{k_{0}}}$ has strictly positive curvature in $\widehat{Y}$. From this, taking sufficiently large $k_{1}$, we can use Theorem 1.4 to get holomorphic sections

$$
V_{1}, \ldots, V_{p+1} \in H^{0}\left(\widehat{\pi^{-1}(Z)}, \mathcal{O}\left(T_{\pi^{-1}(Z)}^{\prime} \otimes\left(\pi^{*} L^{k_{0}} \otimes H_{\mathbb{P}(\mathcal{O}(-L) \oplus \mathcal{O})}\right)^{k_{1}}\right)\right),
$$

which correspond to some basis $V_{1,0}, \ldots, V_{p+1,0}$ of $T_{\pi^{-1}(Z), y_{0}}^{\prime}$. If we use Theorem 1.4 once more and take even larger $k_{1}$, it can be seen that $V_{1}, \ldots, V_{p+1}$ are restrictions of some

$$
\widetilde{V}_{1}, \ldots, \widetilde{V}_{p+1} \in H^{0}\left(\widehat{Y}, \mathcal{O}\left(T_{\widehat{Y}}^{\prime} \otimes\left(\pi^{*} L^{k_{0}} \otimes H_{\mathbb{P}(\mathcal{O}(-L) \oplus \mathcal{O})}\right)^{k_{1}}\right)\right),
$$

which are integrable with respect to $\left(h_{k_{0}} e^{-\psi_{k_{0}}}\right)^{k_{1}}$. Note that $\left.H_{\mathbb{P}(\mathcal{O}(-L) \oplus \mathcal{O})}\right|_{Y}$ is trivial and that $\pi^{*} L=-[X]$ (the dual of the line bundle defined by the divisor $X \subseteq Y)$. Therefore $\widetilde{V}_{1}, \ldots, \widetilde{V}_{p+1}$ can be identified with holomorphic vector fields over $Y$ having zeros of order at least $k_{0} k_{1}$ along $X$. Further, by the integrability condition, we get

$$
\left|\widetilde{V}_{i}\left(x, w_{\alpha}\right)\right| \leqslant C \cdot\left(\left|w_{\alpha}\right|^{k_{0}} \cdot\left|f_{\alpha}(x)\right|^{k_{0}\left(1+k_{0}^{-1 / 2}\right)} \cdot\left|w_{\alpha}\right|\right)^{k_{1}},
$$

hence

$$
\left|\widetilde{V}_{i}\left(x, w_{\alpha}\right)\right| \leqslant C(k) \cdot\left(\left|w_{\alpha}\right| \cdot\left|f_{\alpha}(x)\right|\right)^{\left(k_{0}+1\right) k_{1}} \cdot\left|f_{\alpha}(x)\right|^{k} .
$$

The boundedness of $\left|w_{\alpha}\right| \cdot\left|f_{\alpha}(x)\right|$ in $\left\{\chi_{\psi_{0}} \leqslant 1\right\}$ implies the conclusion. Actually, Lemma 3.6 assures the existence of desired vector fields $V_{i}(1 \leqslant i \leqslant p)$ and one can repeat the proof of Theorem 3.4 in [Ber09].

On the other hand, repeating the proof of the $Z=X$ case in Proposition 3.1 of Ber09 gives the following.

Lemma 3.7. In the situation of Theorem 3.5,

(1) $P_{X \mid Z} \varphi=\iota^{*} \varphi$ a.e. with respect to $\operatorname{MA}\left(P_{X \mid Z} \varphi\right)$.

(2) $P_{X \mid Z} \varphi\left(z_{0}\right)=\iota^{*} \varphi\left(z_{0}\right) \Rightarrow\left(\iota^{*} \theta+d d^{c} \iota^{*} \varphi\right)\left(z_{0}\right) \geqslant 0$.

As a consequence of Theorem 3.5 and Lemma 3.7(1), we obtain the desired representation formula for $\operatorname{MA}\left(P_{X \mid Z} \varphi\right)$ as in the case $Z=X$.

Theorem 3.8. Assume that $Z$ is smooth and $\iota(Z) \nsubseteq \mathbb{B}_{+}(L)$. Then the identity

$$
\operatorname{MA}\left(P_{X \mid Z} \varphi\right)=\mathbf{1}_{\left\{P_{X \mid Z} \varphi=\iota^{*} \varphi\right\}} \cdot\left(\iota^{*} \theta+d d^{c} \iota^{*} \varphi\right)^{p}
$$

holds. Here $\mathbf{1}_{\left\{P_{X \mid Z} \varphi=\iota^{*} \varphi\right\}}$ denotes the characteristic function of the set $\left\{P_{X \mid Z} \varphi=\right.$ $\left.\iota^{*} \varphi\right\}$. In particular, the measure $\mathrm{MA}\left(P_{X \mid Z} \varphi\right)$ has $L^{\infty}$-density with respect to $d \mu$.

3.2. Restricted Bergman kernel asymptotics. From now on, we compare $P_{X \mid Z} \varphi$ with $B_{X \mid Z}(m \varphi)$ in detail. Fix notation as in the previous subsections. In this subsection we always assume that $Z$ is a smooth subvariety of $X$ and that $\iota(Z) \nsubseteq \mathbb{B}_{+}(L)$ holds. First we specify the upper bound of restricted Bergman kernels and show half of our main result. 


\section{Proposition 3.10.}

$$
\limsup _{m \rightarrow \infty} \frac{B_{X \mid Z}(m \varphi)}{m^{p} / p !} d \mu \leqslant \operatorname{MA}\left(P_{X \mid Z} \varphi\right) .
$$

Proof. This is deduced from the two estimates about the upper bound of Bergman kernels. First, we show the so-called Berman's local holomorphic Morse inequality (see [Ber04, Theorem 1.1]) in the restricted case. The proof in the case $Z=X$ is applicable with no change.

Claim 1.

$$
\limsup _{m \rightarrow \infty} \frac{B_{X \mid Z}(m \varphi)}{m^{p} / p !} d \mu \leqslant \mathbf{1}_{\left\{\left(\iota^{*} \theta+d d^{c} \iota^{*} \varphi\right) \geqslant 0\right\}} \cdot\left(\iota^{*} \theta+d d^{c} \iota^{*} \varphi\right)^{p} .
$$

Proof of Claim 1. Fix any $z_{0} \in Z$. If we take an appropriate trivialization patch $U$ around $z_{0}$ with $h_{L, U}\left(z_{0}\right) e^{-\varphi\left(z_{0}\right)}=1$ and denote the eigenvalues of $\iota^{*} \theta+d d^{c} \iota^{*} \varphi$ with respect to the form $\frac{\sqrt{-1}}{2} \sum_{i=1}^{p} d z_{i} \wedge d \bar{z}_{i}$ at $z_{0}$ by $\lambda_{1}, \ldots, \lambda_{p}$, then for an arbitrary section $s \in H^{0}(X \mid Z, \mathcal{O}(m L))$ with $\|s\|_{m \varphi}^{2}=1$, we have

$$
\begin{aligned}
& \frac{\left|s\left(z_{0}\right)\right|_{m \varphi}^{2}}{m^{p} / p !}=\frac{\left|s_{U}\left(z_{0}\right)\right|^{2}}{m^{p} / p !} \\
& \leqslant\left(\int_{|z| \leqslant \frac{\log m}{\sqrt{m}}}\left|s_{U}\right|^{2} e^{-m \sum \lambda_{i}\left|z_{i}\right|^{2}} d \lambda(z)\right)\left(\int_{|z| \leqslant \frac{\log m}{\sqrt{m}}} e^{-m \sum \lambda_{i}\left|z_{i}\right|^{2}} d \lambda(z) \cdot m^{p} / p !\right)^{-1}
\end{aligned}
$$

by the mean value inequality for subharmonic functions. Here $d \lambda$ denotes the Lebesgue measure with respect to $z_{i}$. The $\lim _{\sup } \sin _{m \rightarrow \infty}$ of the numerator in the last side is bounded by $\operatorname{det}_{d \mu} d \lambda\left(z_{0}\right)$, and the denominator behaves as follows if we let $m \rightarrow \infty$ :

$$
\frac{1}{p !} \int_{|w| \leqslant \log m} e^{-\sum \lambda_{i}\left|w_{i}\right|^{2}} d \lambda(w) \rightarrow \begin{cases}\pi^{p} /\left(p ! \lambda_{1} \lambda_{2} \cdots \lambda_{p}\right) & \text { if } \lambda_{i} \geqslant 0 \\ \infty & \text { otherwise. }\end{cases}
$$

(Here we use $w=\sqrt{m} z$ as a new variable.) From this, one can deduce the claim.

The second claim is a direct consequence of the definition of $P_{X \mid Z} \varphi$, and motivates the definition as well.

Claim 2.

$$
\frac{B_{X \mid Z}(m \varphi)}{m^{p} / p !} \leqslant e^{-m\left(\iota^{*} \varphi-P_{X \mid Z} \varphi\right)} \cdot \sup _{Z} \frac{B_{X \mid Z}(m \varphi)}{m^{p} / p !} .
$$

Proof of Claim 2. Note that the supremum on the right-hand side is finite by Claim 1. Fix any $z_{0} \in Z$ and take any $s \in H^{0}(X \mid Z, \mathcal{O}(m L))$ satisfying $\left|s\left(z_{0}\right)\right|_{m \varphi}^{2}=$ $B_{X \mid Z}(m \varphi)\left(z_{0}\right)$ and $\|s\|_{m \varphi}^{2}=1$. Since $|s(z)|_{m \varphi}^{2} \leqslant \sup _{Z} B_{X \mid Z}(m \varphi)$ for any $z \in Z$, we have

$$
\frac{1}{m}\left(\log |s(z)|_{h_{L}^{m}}^{2}-\log \sup _{Z} B_{X \mid Z}(m \varphi)\right) \leqslant \iota^{*} \varphi \text { in } Z .
$$

Since the left-hand side is the pull-back of a $\theta$-psh function on $X$, the above inequality implies

$$
\frac{1}{m}\left(\log |s(z)|_{h_{L}^{m}}^{2}-\log \sup _{Z} B_{X \mid Z}(m \varphi)\right) \leqslant P_{X \mid Z} \varphi \text { in } Z .
$$

Thus Claim 2 is obtained. 
Proposition 3.10 is now easily proved. Actually, Claim 1 and Lemma 3.7(2) imply

$$
\limsup _{m \rightarrow \infty} \frac{B_{X \mid Z}(m \varphi)}{m^{p} / p !} d \mu \leqslant\left(\iota^{*} \theta+d d^{c} \iota^{*} \varphi\right)^{p} \quad \text { in }\left\{P_{X \mid Z} \varphi=\iota^{*} \varphi\right\}
$$

and Claim 2 implies the pointwise convergence

$$
\frac{B_{X \mid Z}(m \varphi)}{m^{p} / p !} \rightarrow 0 \quad(m \rightarrow \infty) \text { in }\left\{P_{X \mid Z} \varphi \neq \iota^{*} \varphi\right\},
$$

so one can conclude Proposition 3.10 by Theorem 3.8 .

Corollary 3.14.

$$
\operatorname{Vol}_{X \mid Z}(L) \leqslant \int_{Z} \operatorname{MA}\left(P_{X \mid Z} \varphi\right) .
$$

Proof. Since $\operatorname{MA}\left(P_{X \mid Z} \varphi\right)$ has $L^{\infty}$-density by Theorem 3.8 we can apply Fatou's lemma to (3.10).

We can now derive the fundamental relation between $P_{X \mid Z} \varphi$ and $B_{X \mid Z}(m \varphi)$.

Theorem 3.15. For every compact set $K \Subset Z \backslash \iota^{-1}\left(\mathbb{B}_{+}(L)\right)$, there exist an integer $m_{0}$ and a positive constant $C \geqslant 0$ such that the inequality

$$
C^{-1} \cdot e^{-m\left(\iota^{*} \varphi-P_{X \mid Z} \varphi\right)} \leqslant B_{X \mid Z}(m \varphi) \leqslant C \cdot m^{p} e^{-m\left(\iota^{*} \varphi-P_{X \mid Z} \varphi\right)}
$$

holds.

Proof. The right-hand side inequality is a direct consequence of Proposition 3.10 and Theorem 3.8. We will show the left-hand side. By the extremal property of the Bergman kernel, it is enough to show the following claim.

Claim. There exist some $m_{0}, C$, and section $s_{m} \in H^{0}(X \mid Z, \mathcal{O}(m L))$ for each $m \geqslant$ $m_{0}$ such that

(1) $\left|s_{m}(z)\right|_{m \psi_{k}}^{2} \geqslant C^{-1}$ for any $z \in K, k \in \mathbb{N}$,

(2) $\left\|s_{m}\right\|_{m \varphi}^{2} \leqslant C$.

Here $\psi_{k} \in \operatorname{PSH}(X, \theta)$ are taken to satisfy $\iota^{*} \psi_{k} \nearrow P_{X \mid Z} \varphi$ a.e. with respect to $d \mu$. Actually, this implies

$$
B_{X \mid Z}(m \varphi) \geqslant \frac{\left|s_{m}(z)\right|_{m \varphi}^{2}}{\left\|s_{m}\right\|_{m \varphi}^{2}} \geqslant C^{-2} e^{-m\left(\iota^{*} \varphi-\iota^{*} \psi_{k}\right)},
$$

so letting $k \rightarrow \infty$ we get the inequality.

Proof of the Claim. Fix $z \in K$. By Kodaira's lemma, we may take some ample $\mathbb{Q}$-divisor $A$ and some effective $\mathbb{Q}$-divisor $E$ on $X$ satisfying $L=A+E$. From this decomposition, we may construct a $\theta$-psh function $\psi_{0}$ with $\psi_{0}^{-1}(-\infty) \subseteq \operatorname{Supp} E$, $\psi_{0} \leqslant \varphi$. Then using Theorem 1.4 twice, we may find suitable $m_{0}, C$ and sections $s_{m} \in H^{0}(X \mid Z, \mathcal{O}(m L))$ for each $m \geqslant m_{0}$ such that

(1) $\left|s_{m}(z)\right|_{\psi_{m, k}}^{2}=1$,

(2) $\left\|s_{m}\right\|_{\psi_{m, k}}^{2} \leqslant C$, 
where $\psi_{m, k}=\left(m-m_{0}\right) \psi_{k}+m_{0} \psi_{0}$. Then we infer

$$
\left\|s_{m}\right\|_{m \varphi}^{2} \leqslant\left\|s_{m}\right\|_{\psi_{m, k}}^{2} \leqslant C,
$$

and since we may assume $e^{m_{0}\left(\varphi-\psi_{0}\right)(z)} \leqslant C$ by the smoothness of $\psi_{0}$ around $z$,

$$
1=\left|s_{m}(z)\right|_{\psi_{m, k}}^{2} \leqslant C\left|s_{m}(z)\right|_{m \varphi_{k}}^{2} .
$$

Here $C$ depends on $m_{0}$ and $K$.

As a consequence of the above results, the sequence of the Monge-Ampère mass of the following Fubini-Study-like potential functions converges to the Monge-Ampère mass of the restricted equilibrium weight. This fact corresponds to the description of restricted volumes via moving intersection numbers (see Theorem 4.6), and has a key role for us to prove the local version of the restricted Fujita approximation in the next subsection. Let us define

$$
u_{m}:=\iota^{*} \varphi+\frac{1}{m} \log B_{X \mid Z}(m \varphi) .
$$

\section{Theorem 3.18.}

$$
u_{m} \rightarrow P_{X \mid Z} \varphi \quad \text { uniformly in any compact subset of } Z \backslash \iota^{-1}\left(\mathbb{B}_{+}(L)\right),
$$

and

$$
\operatorname{MA}\left(u_{m}\right) \rightarrow \operatorname{MA}\left(P_{X \mid Z} \varphi\right) \quad(m \rightarrow \infty)
$$

in the sense of currents.

Proof. The inequality (3.16) is equivalent to

$$
-\frac{\log C}{m}+P_{X \mid Z} \varphi \leqslant \iota^{*} \varphi+\frac{1}{m} \log B_{X \mid Z}(m \varphi) \leqslant \frac{\log C+p \log m}{m}+P_{X \mid Z} \varphi .
$$

This estimate implies that, on any compact subset of $Z \backslash \iota^{-1}\left(\mathbb{B}_{+}(L)\right), u_{m}$ converges uniformly to $P_{X \mid Z} \varphi$. By the continuity property of the Monge-Ampère operator, we deduce

$$
\left(\iota^{*} \theta+d d^{c} u_{m}\right)^{p} \rightarrow\left(\iota^{*} \theta+d d^{c} P_{X \mid Z} \varphi\right)^{p} \quad \text { in } Z \backslash \iota^{-1}\left(\mathbb{B}_{+}(L)\right) .
$$

In particular,

$$
\liminf _{m \rightarrow \infty} \int_{Z \backslash \iota^{-1}\left(\mathbb{B}_{+}(L)\right)}\left(\iota^{*} \theta+d d^{c} u_{m}\right)^{p} \geqslant \int_{Z \backslash \iota^{-1}\left(\mathbb{B}_{+}(L)\right)}\left(\iota^{*} \theta+d d^{c} P_{X \mid Z} \varphi\right)^{p}
$$

holds. Therefore, we only have to show

$$
\limsup _{m \rightarrow \infty} \int_{Z \backslash \iota^{-1}\left(\mathbb{B}_{+}(L)\right)}\left(\iota^{*} \theta+d d^{c} u_{m}\right)^{p} \leqslant \int_{Z \backslash \iota^{-1}\left(\mathbb{B}_{+}(L)\right)}\left(\iota^{*} \theta+d d^{c} P_{X \mid Z} \varphi\right)^{p},
$$

because we already have the current convergence in $Z \backslash \iota^{-1}\left(\mathbb{B}_{+}(L)\right)$, but this is directly seen by Theorem 2.6 and Theorem 3.4 . 
3.3. Restricted Fujita-type approximation. In this subsection, we first give a proof of the restricted Fujita approximation theorem and then finish the poof of Theorem 1.2 .

Theorem 3.19 ([Tak06, Theorem 3.1], ELMNP09, Theorem 2.13]). Let $X$ be a smooth projective variety, $\iota: Z \hookrightarrow X$ a subvariety, and $L$ a big line bundle on $X$. Then for an arbitrary $\varepsilon>0$, the following diagram is commutative, where $\pi_{Z}, \pi_{X}$ are modifications and $\widetilde{Z}, \widetilde{X}$ are smooth such that

(1) in the sense of linear equivalence between $\mathbb{Q}$-divisors, $\pi_{X}^{*} L=A+E$ holds for some semiample and big divisor $A$ and effective divisor $E$, and

(2) $\operatorname{Vol}_{\tilde{X} \mid \widetilde{Z}}(A) \leqslant \operatorname{Vol}_{X \mid Z}(L) \leqslant \operatorname{Vol}_{\widetilde{X} \mid \widetilde{Z}}(A)+\varepsilon$ hold.

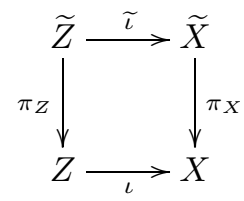

Remark 3.20. By the continuity of the restricted volume (see [ELMNP09, Theorem A] or (3.27) ), the divisor $A$ in Theorem 3.19 can be taken to be ample. This is shown as follows. First we get a decomposition of $\mathbb{Q}$-divisor: $A=A_{0}+E_{0}=$ $\left((1-\delta) A+\delta A_{0}\right)+\left(\delta E_{0}\right)$ by Kodaira's lemma. Then $A_{\delta}:=(1-\delta) A+\delta A_{0}$ is ample and letting $\delta \rightarrow 0, \operatorname{Vol}_{X \mid Z}\left(A_{\delta}\right)$ approximates $\operatorname{Vol}_{X \mid Z}(A)$. From this, it follows that

$$
\operatorname{Vol}_{X \mid Z}(L)=\lim _{m \rightarrow \infty} \frac{\operatorname{dim} H^{0}(X \mid Z, \mathcal{O}(m L))}{m^{p} / p !}
$$

holds. Indeed one can reduce this to the case when $L$ is ample. Since the Serre vanishing theorem forces $H^{0}(X \mid Z, \mathcal{O}(m L))=H^{0}(Z, \mathcal{O}(m L))$, in this case, we may assume $Z=X$. Then (3.21) is obtained from the Riemann-Roch theorem.

Although a proof of Theorem 3.19 has already been obtained in Tak06 or ELMNP09, we have to reprove this to show the local version (Theorem 1.2) at the same time. Our proof of Theorem 3.19] is essentially the same as the proof in Tak06] or [ELMNP09, but we need a more direct proof and do not use a characterization of restricted volumes via multiplier ideal sheaves. We need the "uniformly globally generation theorem", which was first proved in Siu98. It can also be obtained as a corollary of Theorem 1.4

Proposition 3.22 ([Siu98, Proposition 1]). Given a smooth projective variety $X$, there exists a line bundle $G$ such that for any pseudo-effective line bundle $F$ on $X$ with a singular Hermitian metric $h_{F} e^{-\psi}$ whose Chern curvature current is positive, the sheaf $\mathcal{O}(F+G) \otimes \mathcal{I}(\psi)$ is globally generated.

We also need the following lemma, which can be shown by simple algebraic computations. For a proof, see, e.g., [Laz04, 2.2.C].

Lemma 3.23. For an arbitrary line bundle $G$ on $X$ and a positive number $\varepsilon>0$, there exist a subsequence $\left\{\ell_{k}\right\}(k=1,2, \ldots)$ and an integer $m_{0}$ such that

$$
\frac{\operatorname{dim} H^{0}\left(X \mid Z, \mathcal{O}\left(\ell_{k}(m L-G)\right)\right)}{\ell_{k}^{p} / p !} \geqslant m^{p}\left(\operatorname{Vol}_{X \mid Z}(L)-\varepsilon\right)
$$

for any $m \geqslant m_{0}$. 
Proof of Theorem 3.19. Throughout this proof, we fix some $G$ which appeared in Theorem 3.22 and a smooth metric $h_{G}$ on $G$. For any fixed integer $m$, we define the weight of $h_{L}^{m} h_{G}^{-1}$ as

$$
u_{m}:=\varphi+\frac{1}{m} \log \left(\left|s_{m, 1}\right|_{m \varphi}^{2}+\cdots+\left|s_{m, N(m)}\right|_{m \varphi}^{2}\right),
$$

where $\left\{s_{m, 1}, \ldots, s_{m, N(m)}\right\}$ is a complete orthonormal system of $H^{0}(X, \mathcal{O}(m L-G))$ with respect to the norm

$$
\begin{gathered}
\|s\|_{m \varphi}^{2}:=\int_{X}|s|_{m \varphi}^{2} d \mu, \\
|s|_{m \varphi}^{2}:=\left(h_{L}^{m} h_{G}^{-1}\right)(s, s) e^{-m \varphi} .
\end{gathered}
$$

This is essentially the same as $u_{m}$ in Theorem 3.18 ( $\iota=\mathrm{id}$ case). In fact, as in subsection 3.2 , we can get

$$
\left\langle\left(\iota^{*} T_{m}\right)^{p}\right\rangle \rightarrow\left\langle\left(\iota^{*} T\right)^{p}\right\rangle,
$$

where $T_{m}:=\theta+d d^{c} u_{m}, T:=\theta+d d^{c} P_{X} \varphi$ assuming that $\iota$ is a closed embedding and that $Z$ is smooth, $\iota(Z) \nsubseteq \mathbb{B}_{+}(L)$. Here the difference caused by $G$ does not matter, for $G$ has no contribution to the asymptotic behavior of $H^{0}(X, \mathcal{O}(m L-G))$ thanks to the bigness of $L$.

If we set $\mathcal{J}$ as the ideal sheaf generated locally by $s_{m, 1}, \ldots, s_{m, N(m)}$, then $\mathcal{J} \subseteq \mathcal{I}\left(m u_{m}\right)$ holds. Therefore, by taking a log resolution, we have the following commutative diagram, where $\pi_{Z}^{*}$ and $\pi_{X}^{*}$ are modifications from smooth projective varieties such that

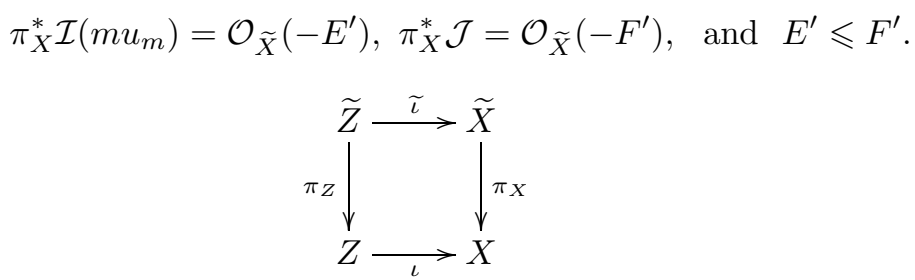

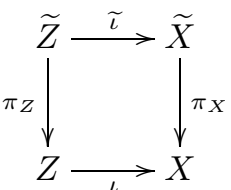

Moreover, since $T_{m}$ has algebraic singularities, we may assume

$$
\pi_{X}^{*} T_{m}=\gamma+[F],
$$

where $\gamma$ is a smooth semipositive form and $F:=F^{\prime} / m$. $[F]$ denotes the closed positive $(1,1)$-current defined by $F$. We claim that this diagram actually satisfies the condition in Theorem 3.19 for a sufficiently large $m$.

By Proposition 3.22, $\mathcal{O}(m L) \otimes \mathcal{I}\left(m u_{m}\right)$ is globally generated. Therefore its pullback $\mathcal{O}\left(m \pi_{X}^{*} L-E^{\prime}\right)$ is also globally generated. For this reason, we may have a semiample divisor $A^{\prime}$ satisfying $m \pi_{X}^{*} L=A^{\prime}+E^{\prime}$. Then the subadditivity property of multiplier ideal sheaves (see [Laz04, 9.5.B]) implies

$$
\begin{aligned}
& H^{0}\left(\widetilde{X} \mid \widetilde{Z}, \mathcal{O}\left(\ell A^{\prime}\right)\right)=H^{0}\left(\widetilde{X} \mid \widetilde{Z}, \mathcal{O}\left(\ell\left(m \pi_{X}^{*} L-E^{\prime}\right)\right)\right) \\
& =H^{0}\left(\widetilde{X} \mid \widetilde{Z}, \pi_{X}^{*}\left(\mathcal{O}(\ell m L) \otimes \mathcal{I}\left(m u_{m}\right)^{\ell}\right)\right) \supseteq H^{0}\left(\widetilde{X} \mid \widetilde{Z}, \pi_{X}^{*}\left(\mathcal{O}(\ell m L) \otimes \mathcal{I}\left(\ell m u_{m}\right)\right)\right),
\end{aligned}
$$

and we get

$$
\begin{aligned}
H^{0}\left(\widetilde{X} \mid \widetilde{Z}, \pi_{X}^{*}\left(\mathcal{O}(\ell m L) \otimes \mathcal{I}\left(\ell m u_{m}\right)\right)\right) & \supseteq H^{0}\left(X \mid Z, \mathcal{O}(\ell m L) \otimes \pi_{X *} \pi_{X}^{*} \mathcal{I}\left(\ell m u_{m}\right)\right) \\
& =H^{0}\left(X \mid Z, \mathcal{O}(\ell m L) \otimes \mathcal{I}\left(\ell m u_{m}\right)\right)
\end{aligned}
$$


by the integral closedness of $\mathcal{I}\left(\ell m u_{m}\right)$. Further,

$$
\begin{aligned}
H^{0}\left(X \mid Z, \mathcal{O}(\ell m L) \otimes \mathcal{I}\left(\ell m u_{m}\right)\right) & \supseteq H^{0}\left(X \mid Z, \mathcal{O}(\ell(m L-G)) \otimes \mathcal{I}\left(\ell m u_{m}\right)\right) \\
& =H^{0}(X \mid Z, \mathcal{O}(\ell(m L-G)))
\end{aligned}
$$

by the definition of $u_{m}$. Consequently, with Lemma 3.23 , it can be seen that there exists a subsequence $\left\{\ell_{k}\right\}$ and a sufficiently large $m$ such that

$$
\frac{\operatorname{dim} H^{0}\left(\widetilde{X} \mid \widetilde{Z}, \mathcal{O}\left(\ell_{k} A^{\prime}\right)\right)}{\ell_{k}^{p} / p !} \geqslant m^{p}\left(\operatorname{Vol}_{X \mid Z}(L)-\varepsilon\right) .
$$

Setting $A:=A^{\prime} / m, E:=E^{\prime} / m$, by homogeneity of restricted volume (ELMNP09, Lemma 2.2]),

$$
\operatorname{Vol}_{\widetilde{X} \mid \widetilde{Z}}(A) \geqslant \operatorname{Vol}_{X \mid Z}(L)-\varepsilon
$$

and $\pi_{X}^{*} L=A+E$ hold. From this estimate it is also possible to deduce that $A$ is big for a sufficiently large $m$, because the above diagram for $\iota$ is also valid for the identity map. The proof of the reversed inequality is not hard.

With the proof of Theorem 3.19, we finally get to our goal of this subsection. Observe that one can approximate $\mathrm{MA}\left(P_{X \mid Z} \varphi\right)$ and $\operatorname{Vol}_{X \mid Z}(L)$ at the same time taking suitable modifications.

Proof of Theorem 1.2. Since $\iota$ is a closed embedding and $\iota(Z) \nsubseteq \mathbb{B}_{+}(L)$, we may assume $\widetilde{\iota}$ is also an embedding and $\widetilde{\iota}(\widetilde{Z}) \nsubseteq \mathbb{B}_{+}\left(A^{\prime}\right)$. By the semiampleness and the bigness of $A^{\prime}$, there exists a smooth semipositive form $\theta_{A}$ in $c_{1}(A)$ such that

$$
\begin{aligned}
\operatorname{Vol}_{\widetilde{X} \mid \widetilde{Z}}(A) & =\operatorname{Vol}_{\widetilde{Z}}\left(\widetilde{\iota}^{*} A\right)=\int_{\widetilde{Z}}\left(\widetilde{\iota}^{*} \theta_{A}\right)^{p} \\
& =\int_{\widetilde{Z}}\left\langle\left(\widetilde{\iota}^{*}\left(\theta_{A}+[E]\right)\right)^{p}\right\rangle .
\end{aligned}
$$

The last equality is a consequence of the non-pluripolarity of the Monge-Ampère product. $\widetilde{\iota}^{*}\left(\theta_{A}+[E]\right)$ and $\widetilde{\iota}^{*}(\gamma+[F])$ are in the same class so that one can apply Theorem 2.6 to (3.25) to deduce the following:

$$
\begin{aligned}
\int_{\widetilde{Z}}\left\langle\left(\widetilde{\iota}^{*}\left(\theta_{A}+[E]\right)\right)^{p}\right\rangle & \geqslant \int_{\widetilde{Z}}\left\langle\left(\widetilde{\iota}^{*}(\gamma+[F])\right)^{p}\right\rangle \\
& =\int_{\widetilde{Z}}\left\langle\left(\pi_{Z}^{*} \iota^{*} T_{m}\right)^{p}\right\rangle=\int_{Z}\left\langle\left(\iota^{*} T_{m}\right)^{p}\right\rangle .
\end{aligned}
$$

Note that we may assume $\widetilde{Z} \nsubseteq \operatorname{Supp} F$ since $\mathbb{B}_{+}(L)=\mathbb{B}\left(L-\frac{1}{m} G\right)$ for any sufficiently large $m$. For an arbitrary $\varepsilon>0$, the proof of Theorem 3.18 shows

$$
\int_{Z}\left\langle\left(\iota^{*} T_{m}\right)^{p}\right\rangle \geqslant \int_{Z}\left\langle\left(\iota^{*} T\right)^{p}\right\rangle-\varepsilon
$$

if we take $m$ sufficiently large. This implies

$$
\operatorname{Vol}_{X \mid Z}(L) \geqslant \operatorname{Vol}_{\widetilde{X} \mid \widetilde{Z}}(A) \geqslant \int_{Z} \operatorname{MA}\left(\iota^{*} P_{X} \varphi\right),
$$

so combining this inequality with Theorem 3.4 and Corollary 3.14 , we finally get the identity

$$
\operatorname{Vol}_{X \mid Z}(L)=\int_{Z} \operatorname{MA}\left(P_{X \mid Z} \varphi\right) .
$$


With this identity and Proposition 3.10. Theorem 1.2 is now concluded from Lemma 2.2 in Ber06, which is shown by basic measure theory.

\section{INTEGRAL REPRESENTATIONS FOR THE RESTRICTED VOLUME}

In this section, we discuss several integral representations of the restricted volume.

Theorem 4.1. Let $Z \subseteq X$ be a (possibly singular) subvariety of $X$ and assume $\iota(Z) \nsubseteq \mathbb{B}_{+}(L)$. Then the following holds:

$$
\begin{aligned}
& \operatorname{Vol}_{X \mid Z}(L)=\int_{Z_{\text {reg }}} \operatorname{MA}\left(P_{X \mid Z_{\text {reg }}} \varphi\right)=\int_{Z_{\text {reg }}} \operatorname{MA}\left(\left(\left.\iota\right|_{Z_{\mathrm{reg}}}\right)^{*} P_{X} \varphi\right) \\
& \quad=\sup _{T} \int_{Z_{\mathrm{reg}}}\left\langle\left(\left(\left.\iota\right|_{Z_{\mathrm{reg}}}\right)^{*} T\right)^{p}\right\rangle=\int_{Z_{\mathrm{reg}}}\left\langle\left(\left(\left.\iota\right|_{Z_{\mathrm{reg}}}\right)^{*} T_{\mathrm{min}}\right)^{p}\right\rangle=\int_{X \backslash \mathbb{B}_{+}(L)}\left(T_{\mathrm{min}}\right)^{p} \wedge[Z],
\end{aligned}
$$

where $T$ runs through all the closed positive currents in $c_{1}(L)$, with small unbounded loci not contained in $\iota(Z)$. We denote by $T_{\min }$ a minimum singular current in $c_{1}(L)$ and denote by $Z_{\mathrm{reg}}$ the regular locus of $Z$. The last integrand is defined as a closed positive current on $X \backslash \mathbb{B}_{+}(L)$ in the manner of Bedford and Taylor, and $[Z]$ denotes the closed positive current defined by $Z$.

Proof. First assume $Z$ is smooth. The first two identities are nothing but (3.27) and Theorem 3.4. The second two are consequences of Theorem 2.6. Let us prove the last identity. Note that the trivial extension of the current $\left(T_{\min }\right)^{p} \wedge[Z]$ to $X$ is a closed positive current and has finite mass by Skoda's extension theorem. Fix a Borel function $\psi$ such that $T_{\min }=\theta+d d^{c} \psi$. By induction on $p$, we are going to prove that

$$
\int_{X \backslash \mathbb{B}_{+}(L)} \rho\left(\theta+d d^{c} \psi\right)^{p} \wedge[Z]=\int_{Z \backslash \iota^{-1}\left(\mathbb{B}_{+}(L)\right)} \iota^{*} \rho\left(\iota^{*} \theta+d d^{c} \psi\right)^{p}
$$

for any Borel function $\rho$ on $X$. The case $p=0$ is trivial. Assume this is true for $p-1$. First fix a smooth function $\rho$ on $Z$. Take some $\chi_{k} \in C_{0}^{\infty}\left(X \backslash \mathbb{B}_{+}(L)\right)$ $(k=1,2,3, \ldots)$ such that $\chi_{k} \equiv 1$ outside of the $1 / k$-neighborhood of $\mathbb{B}_{+}(L)$. Then

$$
\begin{aligned}
& \int_{X \backslash \mathbb{B}_{+}(L)} \chi_{k} \rho\left(\theta+d d^{c} \psi\right) \wedge\left(\theta+d d^{c} \psi\right)^{p-1} \wedge[Z] \\
& =\int_{X \backslash \mathbb{B}_{+}(L)} \chi_{k} \rho \theta \wedge\left(\theta+d d^{c} \psi\right)^{p-1} \wedge[Z]+\psi d d^{c}\left(\chi_{k} \rho\right) \wedge\left(\theta+d d^{c} \psi\right)^{p-1} \wedge[Z] \\
& =\int_{Z \backslash \iota^{-1}\left(\mathbb{B}_{+}(L)\right)} \iota^{*}\left(\chi_{k} \rho \theta\right) \wedge\left(\iota^{*} \theta+d d^{c} \iota^{*} \psi\right)^{p-1}+\iota^{*} \psi d d^{c} \iota^{*}\left(\chi_{k} \rho\right) \wedge\left(\iota^{*} \theta+d d^{c} \iota^{*} \psi\right)^{p-1}
\end{aligned}
$$

by the induction hypothesis. This is equal to

$$
\int_{Z \backslash \iota^{-1}\left(\mathbb{B}_{+}(L)\right)} \iota^{*}\left(\chi_{k} \rho \theta\right) \wedge\left(\iota^{*} \theta+d d^{c} \iota^{*} \psi\right)^{p-1}+\iota^{*}\left(\chi_{k} \rho\right) d d^{c} \iota^{*} \psi \wedge\left(\iota^{*} \theta+d d^{c} \iota^{*} \psi\right)^{p-1} .
$$

Letting $k \rightarrow \infty$, we get (4.2) by the Lebesgue convergence theorem. The general case follows from the density.

The assumption $Z$ is smooth can be dropped if we consider a resolution of singularities, because the Monge-Ampère measure has no mass on any closed proper algebraic subset. For instance, let us prove the first identity. The definition of $P_{X \mid Z_{\text {reg }}} \varphi$ is the same as (3.17). If we take a resolution of singularities, $\operatorname{Vol}_{X \mid Z}(L)=$ 
$\operatorname{Vol}_{\widetilde{X} \mid \widetilde{Z}}\left(\pi_{Z}^{*} L\right)$ holds. It is enough to show that $P_{\widetilde{X} \mid \widetilde{Z}} \pi_{Z}^{*} \varphi=\pi_{Z}^{*} P_{X \mid Z_{\text {reg }}} \varphi$ in the regular locus of $\pi_{Z} \cdot P_{\widetilde{X} \mid \widetilde{Z}} \pi_{Z}^{*} \varphi \leqslant \pi_{Z}^{*} P_{X \mid Z_{\text {reg }}} \varphi$ is trivial and the converse inequality follows by the Riemann-type extension theorem for psh functions. Other identities above are shown in the same manner.

Remark 4.3. The assumption that $T$ has a small unbounded locus can be dropped since we may define the non-pluripolar Monge-Ampère product for any $\theta$-psh function and approximate it by the sequence of minimal singular $\theta$-psh (as in the proof of Proposition 1.20 in BEGZ10]).

The last representation shows that $\operatorname{Vol}_{X \mid Z}(L)$ is independent of $L$ in the same first Chern class. This result was already proved in [ELMNP09] algebraically.

Corollary 4.4. $\operatorname{Vol}_{X \mid Z}(L)$ is determined only by $Z \subseteq X$ and $c_{1}(L)$.

Further, these representations of the restricted volume do not need sections of $L$, hence we can extend the definition of restricted volumes to any class.

Definition 4.5. For any big class $\alpha \in H^{1,1}(X ; \mathbb{R})$ and subvariety $Z \subseteq X$, we define the restricted volume as follows:

$$
\begin{aligned}
& \operatorname{Vol}_{X \mid Z}(\alpha):=\int_{Z_{\mathrm{reg}}} \operatorname{MA}\left(P_{X \mid Z_{\mathrm{reg}}} \varphi\right)=\int_{Z_{\mathrm{reg}}} \operatorname{MA}\left(\left(\left.\iota\right|_{Z_{\mathrm{reg}}}\right)^{*} P_{X} \varphi\right) \\
& =\int_{Z_{\mathrm{reg}}}\left\langle\left(\left(\left.\iota\right|_{Z_{\mathrm{reg}}}\right)^{*} T_{\mathrm{min}}\right)^{p}\right\rangle=\sup _{T} \int_{Z_{\mathrm{reg}}}\left\langle\left(\left(\left.\iota\right|_{Z_{\mathrm{reg}}}\right)^{*} T\right)^{p}\right\rangle=\int_{X \backslash \mathbb{B}_{+}(\alpha)}\left(T_{\mathrm{min}}\right)^{p} \wedge[Z],
\end{aligned}
$$

where $T$ runs through all the closed positive currents in $\alpha$, with small unbounded loci not contained in $\iota(Z)$.

For the definitions of bigness and the augmented base locus for an arbitrary class, see BEGZ10. Note that the regularity of $P_{X} \varphi$ for a general class $\alpha$ is already shown in BD09. We will prove the regularity of $P_{X \mid Z} \varphi$ for the class $c_{1}(L)$ in Section 5. But for a general $\alpha$, the corresponding regularity result seems unknown. The second identity in the above definition is true since it is easily seen that $P_{X \mid Z} \varphi$ has a small unbounded locus even in this case, and the proof of Theorem 3.4 is still valid. The other identities can be proved in the same way as in the case $\alpha=c_{1}(L)$.

At the end of this subsection, we give the representation of restricted volumes via so-called moving intersection number. By definition, the moving intersection number counts the number of points where $Z$ and a general divisor $D \in|m L|$ intersects outside of the base locus. We denote it by $\left\langle(m L)^{p}, Z\right\rangle$. It is already known that $\operatorname{Vol}_{X \mid Z}(L)=\lim _{m \rightarrow \infty} m^{-p}\left\langle(m L)^{p}, Z\right\rangle$ (see [ELMNP09, Theorem 2.13]). The refinement of this result is now obtained.

Theorem 4.6. In the situation of Theorem 4.1,

$$
\begin{aligned}
\operatorname{Vol}_{X \mid Z}(L) & =\lim _{m \rightarrow \infty} \int_{Z} \operatorname{MA}\left(u_{m}\right) \\
& =\lim _{m \rightarrow \infty} \frac{\left\langle(m L)^{p}, Z\right\rangle}{m^{p}}=:\left\|L^{p} . Z\right\| .
\end{aligned}
$$

Proof. The first identity is a direct consequence of Theorem 3.18, The second is easily seen by taking a $\log$ resolution of $|m L|$. In fact, the second identity holds before taking a limit. Notation in the third identity follows from [ELMNP09]. 


\section{5. $L^{2}$-EXTENSION THEOREM FROM A SUBVARIETY}

In this section, we state the desired $L^{2}$-extension theorem for our purpose and give a proof.

Let us first fix notation. Given a holomorphic Hermitian vector bundle $E$ with a metric $h_{E}$ on a Kähler manifold $X$, we denote its Chern curvature tensor by $c(E)$. That is, $c(E):=\sqrt{-1} D^{2}$, where $D$ denotes the exterior covariant derivative associated to the Chern connection of $\left(E, h_{E}\right) . \quad c(E)$ is an $E^{*} \otimes E$-valued real $(1,1)$-form and defines a Hermitian form on $T_{X, x} \otimes E_{x} \quad(x \in X)$ as follows:

$$
H\left(t_{1} \otimes e_{1}, t_{2} \otimes e_{2}\right):=\left(c(E)\left(t_{1}, \sqrt{-1} t_{2}\right) e_{1} \mid e_{2}\right) \quad \text { for } t_{1}, t_{2} \in T_{X, x}, e_{1}, e_{2} \in E_{x} .
$$

Here $(\mid)$ is defined by $h_{E}$. Recall that $c(E)$ is said to be semipositive in the sense of Nakano if $H$ is semipositive everywhere in $X$, and we denote it by $c(E) \geqslant_{\mathrm{Nak}} 0$. If a Kähler metric $\omega$ is fixed, $c(E)$ also defines a Hermitian form on $\left(\bigwedge^{p \cdot q} T_{X, x}^{*}\right) \otimes E_{x}$ as follows:

$$
\theta(\alpha, \beta):=([c(E), \Lambda] \alpha \mid \beta) \text { for } \alpha, \beta \in\left(\bigwedge^{p, q} T_{X, x}^{*}\right) \otimes E_{x} \quad(x \in X),
$$

where $\Lambda$ denotes the formal adjoint operator of the multiplication by $\omega$. It is known that if $p=n$ and $c(E)$ is semipositive in the sense of Nakano, $\theta$ defines a semipositive Hermitian form. We will use the following norm:

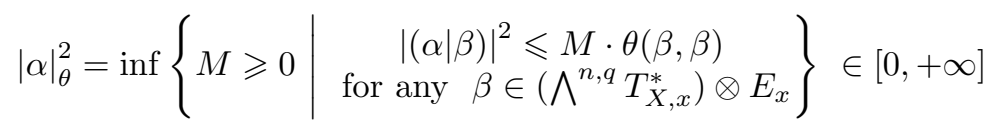

for $\alpha \in\left(\bigwedge^{n, q} T_{X, x}^{*}\right) \otimes E_{x}$.

Theorem 5.1. Let $Z$ be a p-dimensional submanifold of an $n$-dimensional Kähler manifold $X$ with its Kähler form $\omega, K$ a compact subset of $X$. Then there exist constants $N=N(Z, K)>0$ and $C=C(Z, K)>0$ such that the following holds.

Fix any complete Kähler open set $\Omega \subseteq X$ contained in $K$, a holomorphic vector bundle $E \rightarrow X$ with a smooth Hermitian metric $h_{E}$ whose Chern curvature satisfies

$$
c(E) \geqslant_{\mathrm{Nak}} N \cdot \operatorname{id}_{E} \quad \text { on } \Omega,
$$

and $f \in H^{0}\left(Z \cap \Omega, \mathcal{O}\left(K_{X} \otimes E\right)\right)$. Then we have a section $F \in H^{0}\left(\Omega, \mathcal{O}\left(K_{X} \otimes E\right)\right)$ which satisfies $\left.F\right|_{Z \cap \Omega}=f$ and

$$
\int_{\Omega}|F|_{h_{E}}^{2} d V_{\omega, X} \leqslant C \int_{Z \cap \Omega}|f|_{h_{E}}^{2} d V_{\omega, Z}
$$

Although the following proof of this theorem is almost the same as the proof of the Ohsawa-Takegoshi-Manivel $L^{2}$-extension theorem in [Dem00, we describe it for the reader's convenience. The difference from Dem00 is that we deal with arbitrary submanifolds and general vector bundles while we give up sharp estimates.

Proof. There exists some $G \in C^{\infty}\left(\Omega, K_{X} \otimes E\right)$ such that

$$
\left.G\right|_{Z \cap \Omega}=f,\left.\quad(\bar{\partial} G)\right|_{Z \cap \Omega}=0 .
$$

Fix a smooth cut-off function $\rho: \mathbb{R} \rightarrow[0,1]$ satisfying

$$
\rho(t):=\left\{\begin{array}{ll}
1 & \left(t \leqslant \frac{1}{2}\right) \\
0 & (t \geqslant 1)
\end{array} \quad\left|\rho^{\prime}\right| \leqslant 3\right.
$$


Then we set

$$
\begin{aligned}
& G_{\varepsilon}:=\rho\left(\frac{e^{\psi}}{\varepsilon}\right) \cdot G, \\
& g_{\varepsilon}:=\bar{\partial} G_{\varepsilon}=\underbrace{\left(1+\frac{e^{\psi}}{\varepsilon}\right) \rho^{\prime}\left(\frac{e^{\psi}}{\varepsilon}\right) \bar{\partial} \psi_{\varepsilon} \wedge G}_{g_{\varepsilon}^{(1)}}+\underbrace{\rho\left(\frac{e^{\psi}}{\varepsilon}\right) \bar{\partial} G}_{g_{\varepsilon}^{(2)}},
\end{aligned}
$$

where

$$
\begin{aligned}
& \psi_{\varepsilon}:=\log \left(\varepsilon+e^{\psi}\right) \quad\left(\Leftrightarrow 1+\frac{e^{\psi}}{\varepsilon}=\frac{e^{\psi_{\varepsilon}}}{\varepsilon}\right), \\
& \psi:=\log \sum_{\alpha} \chi_{\alpha}^{2} \sum_{i=p+1}^{n}\left|z_{\alpha, i}\right|^{2}, \quad \varepsilon>0 .
\end{aligned}
$$

Here we choose a locally finite system of local coordinates $\left\{z_{\alpha, 1}, \ldots, z_{\alpha, n}\right\}_{\alpha}$ so that

$$
Z \cap U_{\alpha}=\left\{z_{\alpha, p+1}=\cdots=z_{\alpha, n}=0\right\}
$$

hold, and we choose a smooth function $\chi_{\alpha}$ so that the following holds:

$$
\operatorname{Supp} \chi_{\alpha} \subseteq U_{\alpha}, \quad \sum_{\alpha} \chi_{\alpha}^{2}>0, \quad \text { and } \quad \sum_{\alpha} \chi_{\alpha}^{2} \sum_{i=p+1}^{n}\left|z_{\alpha, i}\right|^{2}<e^{-1} \quad \text { in } X .
$$

This $\psi$ satisfies the following conditions (see [Dem82, Proposition 1.4]):

(1) $\psi \in C^{\infty}(X \backslash Z) \cap L_{\text {loc }}^{1}(X)$

$\psi<-1$ in $X, \psi \rightarrow-\infty$ around $Z$.

(2) $e^{-(n-p) \psi}$ is not integrable around any point of $Z$.

(3) There exists a smooth real $(1,1)$-form $\gamma$ in $X$ such that $\sqrt{-1} \partial \bar{\partial} \psi \geqslant \gamma$ holds in $X \backslash Z$.

If the equation

$$
\left\{\begin{array}{l}
\bar{\partial} u_{\varepsilon}=\bar{\partial} G_{\varepsilon} \text { in } \Omega \\
\left|u_{\varepsilon}\right|^{2} e^{-(n-p) \psi} \text { is locally integrable around } Z
\end{array}\right.
$$

has been solved, $u_{\varepsilon}=0$ on $Z$ holds by the above condition, hence the sequence $\left\{G_{\varepsilon}-u_{\varepsilon}\right\}_{\varepsilon}$ is expected to converge to what we want. This is our strategy.

To solve $\bar{\partial}$-equations, we quote the following from Dem00].

Theorem 5.2 (Ohsawa's modified $L^{2}$-estimate [Dem00, Proposition 3.1]) . Let $X$ be a complete Kähler manifold with a Kähler metric $\omega$ ( $\omega$ may not be necessarily complete), $E \rightarrow X$ a holomorphic Hermitian vector bundle. Assume that there exist some smooth functions $a, b>0$, and if we set

$$
\begin{aligned}
& c^{\prime}(E):=a \cdot c(E)-\sqrt{-1} \partial \bar{\partial} a-\sqrt{-1} b^{-1} \partial a \wedge \bar{\partial} a, \\
& \theta^{\prime}(\alpha, \beta):=\left(\left[c^{\prime}(E), \Lambda\right] \alpha \mid \beta\right) \text { for } \alpha, \beta \in\left(\bigwedge^{n, q} T_{X, x}^{*}\right) \otimes E_{x} \quad(x \in X),
\end{aligned}
$$

it holds that

$$
\theta^{\prime} \geqslant 0 \text { on }\left(\bigwedge^{n, q} T_{X, x}\right) \otimes E_{x} \quad \text { for any } x \in X .
$$

Then we have the following. 
For any $g \in L^{2}\left(X,\left(\bigwedge^{n, q} T_{X}^{*}\right) \otimes E\right)$ with $\bar{\partial} g=0$ and

$$
\int_{X}|g|_{\theta^{\prime}}^{2} d V_{\omega, X}<+\infty
$$

there exists a section $u \in L^{2}\left(X,\left(\bigwedge^{n, q-1} T_{X}^{*}\right) \otimes E\right)$ with $\bar{\partial} u=g$ such that

$$
\int_{X}(a+b)^{-1}|u|^{2} d V_{\omega, X} \leqslant 2 \int_{X}|g|_{\theta^{\prime}}^{2} d V_{\omega, X} .
$$

Let us go back to the proof of Theorem 5.1] First, we are going to compute

$$
\theta_{\varepsilon}^{\prime}:=\left[a_{\varepsilon}(c(E)+(n-p) \sqrt{-1} \partial \bar{\partial} \psi)-\sqrt{-1} \partial \bar{\partial} a_{\varepsilon}-b_{\varepsilon}^{-1} \sqrt{-1} \partial a_{\varepsilon} \wedge \bar{\partial} a_{\varepsilon}, \Lambda\right],
$$
where $a_{\varepsilon}, b_{\varepsilon}$ will be defined in the following. If we set

$$
a_{\varepsilon}:=\chi_{\varepsilon}\left(\psi_{\varepsilon}\right)>0
$$

for some smooth function $\chi_{\varepsilon}$, it can be computed as

$$
\begin{aligned}
\partial a_{\varepsilon} & =\chi_{\varepsilon}^{\prime}\left(\psi_{\varepsilon}\right) \partial \psi_{\varepsilon}, \\
\sqrt{-1} \partial \bar{\partial} a_{\varepsilon} & =\chi_{\varepsilon}^{\prime}\left(\psi_{\varepsilon}\right) \sqrt{-1} \partial \bar{\partial} \psi_{\varepsilon}+\chi_{\varepsilon}^{\prime \prime}\left(\psi_{\varepsilon}\right) \sqrt{-1} \partial \psi_{\varepsilon} \wedge \bar{\partial} \psi_{\varepsilon} \\
& =\chi_{\varepsilon}^{\prime}\left(\psi_{\varepsilon}\right) \sqrt{-1} \partial \bar{\partial} \psi_{\varepsilon}+\frac{\chi_{\varepsilon}^{\prime \prime}\left(\psi_{\varepsilon}\right)}{\chi_{\varepsilon}^{\prime}\left(\psi_{\varepsilon}\right)^{2}} \sqrt{-1} \partial a_{\varepsilon} \wedge \bar{\partial} a_{\varepsilon},
\end{aligned}
$$

so comparing with (5.3), it is natural to set

$$
b_{\varepsilon}:=-\frac{\chi_{\varepsilon}^{\prime}\left(\psi_{\varepsilon}\right)^{2}}{\chi_{\varepsilon}^{\prime \prime}\left(\psi_{\varepsilon}\right)}(>0) \text {. }
$$

We finally define

$$
\chi_{\varepsilon}(t):=\varepsilon-t+\log (1-t) .
$$

Then for sufficiently small $\varepsilon>0$, we have

$$
\begin{aligned}
& a_{\varepsilon} \geqslant \varepsilon-\log \left(\varepsilon+e^{-1}\right) \geqslant 1 \\
& \sqrt{-1} \partial \bar{\partial} a_{\varepsilon}+b_{\varepsilon}^{-1} \sqrt{-1} \partial a_{\varepsilon} \wedge \bar{\partial} a_{\varepsilon}=\chi_{\varepsilon}^{\prime}\left(\psi_{\varepsilon}\right) \sqrt{-1} \partial \bar{\partial} \psi_{\varepsilon} \leqslant-\sqrt{-1} \partial \bar{\partial} \psi_{\varepsilon},
\end{aligned}
$$

hence

$$
\theta_{\varepsilon}^{\prime} \geqslant\left[c(E)+(n-p) \sqrt{-1} \partial \bar{\partial} \psi+\sqrt{-1} \partial \bar{\partial} \psi_{\varepsilon}, \Lambda\right] .
$$

On the other hand, simple computations show

$$
\begin{aligned}
\partial \psi_{\varepsilon} & =\frac{e^{\psi}}{\varepsilon+e^{\psi}} \partial \psi \\
\sqrt{-1} \partial \bar{\partial} \psi_{\varepsilon} & =\frac{e^{\psi}}{\varepsilon+e^{\psi}} \sqrt{-1} \partial \bar{\partial} \psi+\frac{e^{\psi}}{\varepsilon+e^{\psi}} \sqrt{-1} \partial \psi \wedge \bar{\partial} \psi-\frac{e^{2 \psi}}{\left(\varepsilon+e^{\psi}\right)^{2}} \sqrt{-1} \partial \psi \wedge \bar{\partial} \psi \\
& =\frac{e^{\psi}}{\varepsilon+e^{\psi}} \sqrt{-1} \partial \bar{\partial} \psi+\frac{\varepsilon}{e^{\psi}} \sqrt{-1} \partial \psi_{\varepsilon} \wedge \bar{\partial} \psi_{\varepsilon} .
\end{aligned}
$$

Therefore, by the compactness of $K$, there exists a constant $N(Z, K)>0$ such that

$$
c(E) \geqslant_{\mathrm{Nak}} N \cdot \operatorname{id}_{\mathrm{E}} \quad \text { on } \Omega
$$

implies

$$
\theta_{\varepsilon}^{\prime} \geqslant 0 \text { on }\left(\bigwedge^{n, 1} T_{X, x}^{*}\right) \otimes E_{x} \quad \text { for all } x \in \Omega,
$$

and eigenvalues of $\theta_{\varepsilon}^{\prime}$ are bounded from below by a positive constant (uniformly with respect to $\varepsilon$ ) near $Z \cup \Omega$. 
Next we will estimate $\bar{\partial} \psi_{\varepsilon}$ by $|\cdot|_{\theta_{\varepsilon}^{\prime}}$. Fix arbitrary $\alpha, \beta \in\left(\bigwedge^{n, 1} T_{X, x}^{*}\right) \otimes E_{x}$. By definition,

$$
\left|\bar{\partial} \psi_{\varepsilon} \wedge \alpha\right|_{\theta_{\varepsilon}^{\prime}}^{2}=\inf \left\{\begin{array}{l|l}
M \geqslant 0 \mid & \begin{array}{c}
\left.\left|\bar{\partial} \psi_{\varepsilon} \wedge \alpha\right| \beta\right)\left.\right|^{2} \leqslant M \cdot\left(\left[c_{\varepsilon}^{\prime}(E) \Lambda\right] \beta \mid \beta\right) \\
\text { for any } \beta \in\left(\bigwedge^{n, 1} T_{X, x}^{*}\right) \otimes E_{x}
\end{array}
\end{array}\right\},
$$

so it is enough to estimate $\left|\left(\bar{\partial} \psi_{\varepsilon} \wedge \alpha \mid \beta\right)\right|^{2}$. This can be done as follows:

$$
\begin{aligned}
& \left|\left(\bar{\partial} \psi_{\varepsilon} \wedge \alpha \mid \beta\right)\right|^{2}=\left|\left(\alpha \mid\left(\bar{\partial} \psi_{\epsilon}\right)^{\sharp} \beta\right)\right|^{2} \\
& \leqslant|\alpha|^{2} \cdot\left|\left(\bar{\partial} \psi_{\epsilon}\right)^{\sharp} \beta\right|^{2}=|\alpha|^{2}\left(\left(\bar{\partial} \psi_{\varepsilon}\right)\left(\bar{\partial} \psi_{\varepsilon}\right)^{\sharp} \beta \mid \beta\right)=|\alpha|^{2}\left(\left[\sqrt{-1} \partial \psi_{\epsilon} \wedge \bar{\partial} \psi_{\epsilon}, \Lambda\right] \beta \mid \beta\right)
\end{aligned}
$$

by Shwartz' inequality ( $\sharp$ denotes taking the formal adjoint of the multiplication operator), and the last term is bounded by

$$
\begin{aligned}
& \frac{e^{\psi}}{\varepsilon}|\alpha|^{2}\left(\left[\sqrt{-1} \partial \bar{\partial} \psi_{\varepsilon}-\frac{e^{\psi}}{\varepsilon+e^{\psi}} \sqrt{-1} \partial \bar{\partial} \psi, \Lambda\right] \beta \mid \beta\right) \\
& \leqslant \frac{e^{\psi}}{\varepsilon}|\alpha|^{2}\left(\left[c(E)+(n-p) \sqrt{-1} \partial \bar{\partial} \psi+\sqrt{-1} \partial \bar{\partial} \psi_{\varepsilon}, \Lambda\right] \beta \mid \beta\right) \\
& \leqslant \frac{e^{\psi}}{\varepsilon}|\alpha|^{2}\left(\left[c_{\varepsilon}^{\prime}(E), \Lambda\right] \beta \mid \beta\right) .
\end{aligned}
$$

The last inequality is a consequence of (5.4). Thus, we may get a desired estimate

$$
\left|\bar{\partial} \psi_{\varepsilon} \wedge \alpha\right|_{\theta_{\varepsilon}^{\prime}}^{2} \leqslant \frac{e^{\psi}}{\varepsilon}|\alpha|^{2} .
$$

This time we estimate $g_{\varepsilon}=g_{\varepsilon}^{(1)}+g_{\varepsilon}^{(2)}$. By (5.6) and $\operatorname{Supp} g_{\varepsilon}^{(1)} \subseteq\left\{e^{\psi}<\varepsilon\right\}, g_{\varepsilon}^{(1)}$ can be estimated. Namely,

$$
\int_{\Omega \backslash Z}\left|g_{\varepsilon}^{(1)}\right|_{\theta_{\varepsilon}^{\prime}}^{2} e^{-(n-p) \psi} d V_{\omega, X} \leqslant 4 \int_{\Omega \backslash Z}|G|^{2} \rho^{\prime}\left(\frac{e^{\psi}}{\varepsilon}\right)^{2} e^{-(n-p) \psi} d V_{\omega, X}
$$

holds. Since $e^{\psi} \sim \sum_{i=p+1}^{n}\left|z_{\alpha, i}\right|^{2}$ on $U_{\alpha}$, thanks to the compactness of $K$ we get

$$
\limsup _{\varepsilon \rightarrow 0} \int_{\Omega \backslash Z}\left|g_{\varepsilon}^{(1)}\right|_{\theta_{\varepsilon}^{\prime}}^{2} e^{-(n-p) \psi} d V_{\omega, X} \leqslant C \int_{Z \cap \Omega}|f|^{2} d V_{\omega, Z}<+\infty .
$$

We can also estimate $g_{\varepsilon}^{(2)}$. Note that eigenvalues of $\theta_{\varepsilon}^{\prime}$ are bounded below. Then we get

$$
\int_{\Omega \backslash Z}\left|g_{\varepsilon}^{(2)}\right|_{\theta_{\varepsilon}^{\prime}}^{2} e^{-(n-p) \psi} d V_{\omega, X} \leqslant O(\varepsilon)<+\infty
$$

because we can see that $\left|g_{\varepsilon}^{(2)}\right|_{\theta_{\varepsilon}^{\prime}}^{2}=O\left(e^{\psi}\right)$ holds in $\operatorname{Supp} g_{\varepsilon}^{(2)} \subseteq\left\{e^{\psi}<\varepsilon\right\}$, by $\left.\bar{\partial} G\right|_{Z \cap \Omega}=0$ (using the Taylor expansion).

Now we can apply the modified $L^{2}$-estimate for each $\varepsilon$ in $\Omega \backslash Z$. Note that $\Omega \backslash Z$ is a complete Kähler manifold (see [Dem82, Theorem 1.5]). There exists a sequence $\left\{u_{\varepsilon}\right\} \subseteq L^{2}\left(\Omega, K_{X} \otimes E\right)$ such that

$$
\int_{\Omega \backslash Z}\left(a_{\varepsilon}+b_{\varepsilon}\right)^{-1}\left|u_{\varepsilon}\right|^{2} e^{-(n-p) \psi} d V_{\omega, X} \leqslant 2 \int_{\Omega \backslash Z}\left|g_{\varepsilon}\right|_{\theta_{\varepsilon}^{\prime}}^{2} e^{-(n-p) \psi} d V_{\omega, X}<+\infty
$$

holds. 
Let us estimate the left-hand side of the inequality. It can be easily seen that

$$
\begin{aligned}
& \psi_{\varepsilon} \leqslant \log \left(\varepsilon+e^{-1}\right) \leqslant-1+O(\varepsilon) \\
& a_{\varepsilon} \leqslant(1+O(\varepsilon)) \psi_{\varepsilon}^{2} \\
& b_{\varepsilon}=\left(2-\psi_{\varepsilon}\right)^{2} \leqslant(9+O(\varepsilon)) \psi_{\varepsilon}^{2} \\
& a_{\varepsilon}+b_{\varepsilon} \leqslant(10+O(\varepsilon)) \psi_{\varepsilon}^{2} \leqslant(10+O(\varepsilon))\left(-\log \left(\varepsilon+e^{\psi}\right)\right)^{2}
\end{aligned}
$$

and

$$
\int_{\Omega} \frac{\left|G_{\varepsilon}\right|^{2}}{\left(\varepsilon+e^{\psi}\right)^{(n-p)}\left(-\log \left(\varepsilon+e^{\psi}\right)\right)^{2}} d V_{\omega, X} \leqslant \frac{M}{(\log \varepsilon)^{2}}
$$

hold. Therefore, if we set $F_{\varepsilon}:=G_{\varepsilon}-u_{\varepsilon}$, it follows that

$$
\begin{aligned}
& \limsup _{\varepsilon \rightarrow 0} \int_{\Omega \backslash Z} \frac{\left|F_{\varepsilon}\right|^{2}}{\left(\varepsilon+e^{\psi}\right)^{(n-p)}\left(-\log \left(\varepsilon+e^{\psi}\right)\right)^{2}} d V_{\omega, X} \\
& \leqslant \limsup _{\varepsilon \rightarrow 0}\left(22 \int_{\Omega \backslash Z}\left|g_{\varepsilon}\right|_{\theta_{\varepsilon}^{\prime}}^{2} e^{-(n-p) \psi} d V_{\omega, X}+\frac{2 M}{(\log \varepsilon)^{2}}\right) \\
& \leqslant C \int_{Z \cap \Omega}|f|^{2} d V_{\omega, Z}<+\infty .
\end{aligned}
$$

By construction, $\bar{\partial} F_{\varepsilon}=0$ holds on $\Omega \backslash Z$ and in fact also in $\Omega$, thanks to the Riemann extension theorem.

Finally, let $\varepsilon \searrow 0$. Then after taking a weakly convergent subsequence, we get a $F \in L^{2}\left(\Omega, K_{X} \otimes E\right)$ such that $\bar{\partial} F=0$ in $\Omega$ and

$$
\int_{\Omega} \frac{|F|^{2}}{e^{(n-p) \psi}(-\psi)^{2}} d V_{\omega, X} \leqslant C \int_{Z \cap \Omega}|f|^{2} d V_{\omega, Z}
$$

By the compactness of $K$, we get the conclusion.

Proof of Theorem 1.4. Since $X$ is projective, we may take a global meromorphic section $\sigma$ of $L$ and may assume $\operatorname{Supp}(\operatorname{div}(\sigma)) \cap Z \subseteq Z$. Fix a hypersurface $H \subseteq X$ such that $X \backslash H$ is Stein, $\operatorname{Supp}(\operatorname{div}(\sigma)) \subseteq H$, and $H \cap Z \subsetneq Z$ hold. Then $\left.L\right|_{X \backslash H}$ is trivial so that we may identify $\varphi$ as a psh function on $X \backslash H$.

Let $\psi$ be a smooth exhaustive strictly-psh function in $X \backslash H$ and set $\Omega_{k}:=\{\psi<$ $k\}$. Since $X \backslash H$ is Stein, there exists a sequence $\varphi_{k} \in \operatorname{PSH}\left(\Omega_{k}\right)$ satisfying $\varphi_{k} \searrow \varphi$ (pointwise convergence in $\Omega_{k}$ ). Note that $\varphi_{k}$ does not loss positivity.

We apply Theorem 5.1 to $\Omega^{\prime}:=\Omega_{k}$ and $E^{\prime}:=K_{X}^{-1} \otimes E \otimes L$ for each $k$. Then by assumption there are sections $\widetilde{s}_{k} \in H^{0}\left(\Omega_{k}, \mathcal{O}\left(K_{X} \otimes E^{\prime}\right)\right)$ such that $\left.\widetilde{s}_{k}\right|_{Z \cap \Omega_{k}}=s$ and

$$
\begin{aligned}
\int_{\Omega_{k}}\left|\widetilde{s}_{k}\right|^{2} e^{-\varphi_{k}} d V_{\omega, X} & \leqslant C \int_{Z \cap \Omega_{k}}|s|^{2} e^{-\varphi_{k}} d V_{\omega, Z} \\
& \leqslant C \int_{Z}|s|^{2} e^{-\varphi} d V_{\omega, Z}
\end{aligned}
$$

for a constant $C$. If we fix $l \in \mathbb{N}$, there exists some constant $c(l) \leqslant e^{-\varphi_{l}}$ in $\Omega_{l}$, hence we have

$$
c(l) \cdot \int_{\Omega_{l}}\left|\widetilde{s}_{k}\right|^{2} d V_{\omega, X} \leqslant C \int_{Z}|s|^{2} e^{-\varphi} d V_{\omega, Z}
$$


Using the diagonal process, we may find a subsequence: $\widetilde{s}_{k(i)} \rightarrow \widetilde{s}$ (weakly $L^{2}$ convergent on $X$ ). By Lemma 5.8, $\bar{\partial} \widetilde{s}_{k(i)}=0$ implies that this is actually the pointwise convergence so that $\left.\widetilde{s}\right|_{Z \cap(X \backslash H)}=s$ holds. We can deduce

$$
\int_{X \backslash H}|\widetilde{s}|^{2} e^{-\varphi} d V_{\omega, X} \leqslant C \int_{Z}|s|^{2} e^{-\varphi} d V_{\omega, Z}
$$

by (5.7), and by the lower-semicontinuity of $L^{2}$-norm. Therefore $\widetilde{s}$ can be extended to $X$ by the Riemann extension theorem. Thus we conclude the theorem.

Lemma 5.8. Let $f_{k}, f$ be holomorphic functions defined in a domain $\Omega \subseteq \mathbb{C}^{n}$. Assume that the sequence $\left\{f_{k}\right\}$ weakly $L^{2}$-converges to $f$. Then $\left\{f_{k}\right\}$ converges to $f$ pointwise in $\Omega$.

Proof. Fix any point $x \in \Omega$. Taking $\chi \in C_{0}^{\infty}(\Omega)$ with $\chi \equiv 1$ near $x$, we have

$$
f_{k}(x)=\int_{\zeta \in \Omega} K_{\mathrm{BM}}^{n, 0}(x, \zeta) \wedge \bar{\partial} \chi(\zeta) \wedge f_{k}(\zeta) \rightarrow \int_{\zeta \in \Omega} K_{\mathrm{BM}}^{n, 0}(x, \zeta) \wedge \bar{\partial} \chi(\zeta) \wedge f(\zeta)=f(x)
$$

by the Koppelman formula. Here $K_{\mathrm{BM}}^{p, q}$ denotes the $(p, q)$-part of the BochnerMartinelli kernel.

\section{ACKNOWLEDGMENTS}

The author would like to express his gratitude to his advisor, Professor Shigeharu Takayama, for his warm encouragement and suggestions and for reading the drafts. The author also would like to thank Professor Takeo Ohsawa, Mihai Paun, and Dano Kim for several helpful comments concerning the $L^{2}$-extension theorem (Theorem 1.4). He is indebted to Doctor Shin-ichi Matsumura for valuable discussions at the seminars. This research was supported by JSPS Research Fellowships for Young Scientists (22-6742).

\section{REFERENCES}

[BT76] E. Bedford, A. Taylor, The Dirichlet problem for a complex Monge-Ampère equation. Invent. Math. 37 (1976), no. 1, 1-44. MR0445006 (56:3351)

[Ber04] R. Berman, Bergman kernels and local holomorphic Morse inequalities. Math. Z. 248 (2004), no. 2, 325-344. MR2088931 (2005g:32024)

[Ber06] R. Berman, Super Toeplitz operators on line bundles. J. Geom. Anal. 16 (2006), no. 1, 1-22. MR2211329 (2007f:32027)

[Ber09] R. Berman, Bergman kernels and equilibrium measures for line bundles over projective manifolds. Amer. J. Math. 131 (2009), no. 5, 1485-1524. MR2559862 (2010g:32030)

[BB10] R. Berman, S. Boucksom, Growth of balls of holomorphic sections and energy at equilibrium. Invent. Math. 181 (2010), no. 2, 337-394. MR2657428 (2011h:32021)

[BD09] R. Berman, J. P. Demailly, Regularity of plurisubharmonic upper envelopes in big cohomology classes. Preprint (2009) arXiv:0905.1246.

[Bou02] S. Boucksom, On the volume of a line bundle. Internat. J. Math. 13 (2002), no. 10, 1043-1063. MR.1945706 (2003j:32025)

[BEGZ10] S. Boucksom, P. Eyssidieux, V. Guedj, A. Zeriahi, Monge-Ampère equations in big cohomology classes. Acta Math. 205 (2010), no. 2, 199-262. MR2746347 (2011k:32049)

[Dem82] J. P. Demailly, Estimations sp2 pour l'opérateur $\bar{\partial}$ d'un fibré vectoriel holomorphe semi-positif au-dessus d'une variété kählérienne compléte. Ann. Sci. École Norm. Sup. (4) 15 (1982), no. 3, 457-511. MR690650 (85d:32057) 
[Dem00] J. P. Demailly, On the Ohsawa-Takegoshi-Manivel $L^{2}$ extension theorem. Complex analysis and geometry (Paris, 1997), 47-82, Progr. Math., 188, Birkhäuser, Basel, 2000. MR $1782659(2001 \mathrm{~m}: 32041)$

[ELMNP09] L. Ein, R. Lazarsfeld, M. Mustaţă, M. Nakamaye, M. Popa, Restricted volumes and base loci of linear series. Amer. J. Math. 131 (2009), no. 3, 607-651. MR 2530849 (2010g:14005)

[Fuj94] T. Fujita, Approximating Zariski decomposition of big line bundles. Kodai Math. J. 17 (1994), no. 1, 1-3. MR.1262949 (95c:14053)

[HM06] C. Hacon, J. McKernan, Boundedness of pluricanonical maps of varieties of general type. Invent. Math. 166 (2006), no. 1, 1-25. MR2242631 (2007e:14022)

[Kim10] D. Kim, $L^{2}$ extension of adjoint line bundle sections. Ann. Inst. Fourier (Grenoble) 60 (2010), no. 4, 1435-1477. MR2722247 (2011m:32027)

[Kli91] M. Klimek, Pluripotential theory. London Mathematical Society Monographs. New Series, 6. Oxford Science Publications. The Clarendon Press, Oxford University Press, New York, 1991. MR.1150978(93h:32021)

[Laz04] R. Lazarsfeld, Positivity in algebraic geometry. I, and II. A Series of Modern Surveys in Mathematics, 48, and 49. Springer-Verlag, Berlin, 2004. MR2095471 (2005k:14001a)

[Ohs01] T. Ohsawa, On the extension of $L^{2}$ holomorphic functions. V. Effects of generalization. Nagoya Math. J. 161 (2001), 1-21. MR.1820210(2001m:32011)

[Siu98] Y. Siu, Invariance of plurigenera. Invent. Math. 134 (1998), no. 3, 661-673. MR.1660941 (99i:32035)

[Tak06] S. Takayama, Pluricanonical systems on algebraic varieties of general type. Invent. Math. 165 (2006), no. 3, 551-587. MR.2242627 (2007m:14014)

[Tian90] G. Tian, On a set of polarized Kähler metrics on algebraic manifolds. J. Differential Geom. 32 (1990), no. 1, 99-130. MR1064867 (91j:32031)

[Tsu06] H. Tsuji, Pluricanonical systems of projective varieties of general type. I. Osaka J. Math. 43 (2006), no. 4, 967-995. MR2303558 (2008j:14030)

Graduate School of Mathematical Sciences, The University of Tokyo, 3-8-1 Komaba Meguro-Ku, TOKYo 153-0041, JAPAN

E-mail address: hisamoto@ms.u-tokyo.ac.jp 